\title{
Lagrangian predictability of high-resolution regional models: the special case of the Gulf of Mexico
}

\author{
P. C. Chu ${ }^{1}$, L. M. Ivanov ${ }^{1,2}$, L. H. Kantha ${ }^{3}$, T. M. Margolina ${ }^{2}$, O. V. Melnichenko ${ }^{2}$, and Y. A. Poberezhny ${ }^{2}$ \\ ${ }^{1}$ Naval Ocean Analysis and Prediction Laboratory, Department of Oceanography, Naval Postgraduate School, Monterey, \\ California, 93943, USA \\ ${ }^{2}$ Marine Hydrophysical Institute, the Ukrainian National Academy of Sciences, Kapitanskaya 2, Sevastopol, 99011, Ukraine \\ ${ }^{3}$ University of Colorado, Boulder, Colorado, 80309, USA
}

Received: 7 March 2003 - Revised: 15 July 2003 - Accepted: 22 July 2003 - Published: 25 February 2004

Part of Special Issue "Dedicated to Prof. A. D. Kirwan Jr. on the occasion of his 70th birthday"

\begin{abstract}
The Lagrangian prediction skill (model ability to reproduce Lagrangian drifter trajectories) of the nowcast/forecast system developed for the Gulf of Mexico at the University of Colorado at Boulder is examined through comparison with real drifter observations. Model prediction error (MPE), singular values (SVs) and irreversible-skill time (IT) are used as quantitative measures of the examination. Divergent (poloidal) and nondivergent (toroidal) components of the circulation attractor at $50 \mathrm{~m}$ depth are analyzed and compared with the Lagrangian drifter buoy data using the empirical orthogonal function (EOF) decomposition and the measures, respectively. Irregular (probably, chaotic) dynamics of the circulation attractor reproduced by the nowcast/forecast system is analyzed through Lyapunov dimension, global entropies, toroidal and poloidal kinetic energies. The results allow assuming exponential growth of prediction error on the attractor. On the other hand, the $q$-th moment of MPE grows by the power law with exponent of $3 q / 4$. The probability density function (PDF) of MPE has a symmetrical but nonGaussian shape for both the short and long prediction times and for spatial scales ranging from $20 \mathrm{~km}$ to $300 \mathrm{~km}$. The phenomenological model of MPE based on a diffusion-like equation is developed. The PDF of IT is non-symmetric with a long tail stretched towards large ITs. The power decay of the tail was faster than 2 for long prediction times.
\end{abstract}

\section{Introduction}

During the World Ocean Circulation Experiment (WOCE), the ocean velocity observation has been significantly advanced with extensive spatial and temporal coverage using near-surface Lagrangian drifters, RAFOS floats, and Autonomous Lagrangian Circulation Explorers (ALACEs). Trajectories of these quasi-Lagrangian drifters reflect the

Correspondence to: L. M. Ivanov

(lmivanov@alpha.mhi.iuf.net) whole spectrum of ocean motions, including meso- and submesoscale eddies, various waves, inertial and semidiurnal motions, and provide invaluable resources to estimate the nowcast/forecast skill of high-resolution regional ocean models in terms of model ability to simulate from synoptic to submesoscale eddy movements.

However, direct comparison between model and quasiLagrangian/Lagrangian observations is difficult since neither the dynamics of numerical models nor the model input (bathymetry, external forcing and subscale parameteriztions) are identical to the reality. It needs to be determined if the model-data difference comes from deficiency in modeling ocean physics, from some unessential imperfection, or from unrepresentative data (Davis et al., 1996). It is clear that the high-resolution ocean model and the Lagrangian drifter data should be compared only in the statistical sense.

The commonly used methods for model-data comparison are listed as follows. The first approach estimates the mean pseudo-Eulerian circulation pattern and/or Eulerian statistics from Lagrangian trajectories (e.g. Figueroa and Olson, 1994; Davis et al., 1996; Acero-Schertzer et al., 1997; Stutzer and Krauss, 1998; Garraffo et al., 2001b). The second approach is to compute the Lagrangian statistics for the real drifters and the modeled synthetic particles and then to compare these statistics by way of statistical tests (e.g. Garraffo et al., 2001a; McClean et al., 2002). The third approach focuses on comparison of attractors reproduced by a model and detected from the Lagrangian data (Chu et al., 2002a). The EOF technique is the mathematical tool of this approach. The fourth approach which will be applied here, is to estimate Lagrangian prediciton skill through statistics of nonasymptotic indicators (measures), such as the model prediction error, the finite scale Lyapunov exponent (Lacorata et al., 2001), the singular vectors (Lorenz, 1965), stable and unstable manifolds (Wiggins, 1992; Kuznetsov et al., 2002), the irreversible-skill time (Ivanov et al., 1994; Chu et al., 2002c) and others. Early the terms "time of predictability" and "valid prediction period" were used instead of "the 
irreversible-skill time", hereinafter IT, (Chu et al., 2002a,b). In our opinion the new name "IT", better corresponds to the physical nature of a model prediction skill.

The primary goals of the present paper are:

(i) to understand what quantitative measures may be effectively applied for the analysis of model ability to predict the real drifter dynamics. Such an ability was called "Lagrangian model predictability" by Mariano et al. (2002). Three local (non-asymptotic) measures: the MPE, SVs and IT will be applied,

(ii) to study Lagrangian predictability of a high-resolution regional model through non-asymptotic criteria. We will examine the Gulf of Mexico model with $1 / 12^{\circ}$ horizontal resolution (Kantha et al., 1999; Kantha and Clayson, 2000),

(iii) to find the fundamental statistics of model pediciton skill and parameterize an evolution law for the mean prediction error. That in general allow to detect when model Lagrangian predictability breaks down.

All these problems are in focus of the modern predictability studies of the practical oceanography and meteorology (Robinson et al., 1999; Palmer, 2000). The knowledge of Lagrangian predictability of high-resolution models is very important for the numerous oceanographic and ecological applications because tracer dispersion and pollutant spread are most easily expressed in terms of Lagrangian velocity correlations.

The knowledge of a statistical law governing the average growth of prediction errors, which initially have a given or even the zero value, would allow us to predict the expected model skill and extending over a given period of time in the future.

Note that the high-resolution oceanographic modeling is supported by the argument that the models of high-resolution reproduce the mesoscale eddy dynamics more correctly versus those of eddy-permitting resolution (Smith et al., 1992). Statistics of 3-D mesoscale eddies dynamics reproduced by the high-resolution models with horizontal resolution of 5-10 km can be assessed only through Lagrangian drifter observations.

The outline of this paper is listed as follows. Section 2 discusses the measures of Lagrangian predictability, such as the MPE, SVs and IT. An original approach developed in the present paper for computations of the singular values and singular vectors is adressed in Appendix A.

The spatio-temporal structure of the model attractor is analyzed through Moffat-Zeldovich decomposition (MZD), empirical orthogonal functions and global entropy. This is discussed in Sect. 3.

Using MZD two subspaces generated by the divergent (poloidal) and nondivergent (toroidal) EOFs, respectively, are introduced. Knowledge of EOF decomposition for the circulation allows estimating Lyapunov dimension of the circulation attractor. Section 4 contains the results of such an analysis.
Statistics of the model prediction error and the IT are discussed in Sect. 5. We demonstrate that the process of displacement with time of the real drifters and modeled particles is described by non-Gaussian statistics for both small and large times. The growth of MPE holds the power law. This is a signature of long-term correlations between the prediction and reality. A simple empirical model is proposed for description of MPE growth. We pointed out that linear-tangent models of MPE evolution are not sufficient for the analysis of MPE in the Gulf of Mexico circulation model.

Section 6 develops and illustrates a new approach for the reconstruction of circulation from rare Lagrangian observations. The last section is a summary and discussion.

\section{Measures of Lagrangian predictability}

\subsection{Local Lagrangian predictability}

We may speculate three possible scenarios of Lagrangian predictability. First, the model reproduces the pattern of the real circulation attractor including its small-scale details and predicts a drifter behavior for long times (the global Lagrangian predictability). Second, in general the circulation attractor is correctly reproduced but not all the small-scale details of the circulation topology are resolved. Here, the predictability of the drifter dynamics exists only for the short and intermediate time intervals (the local Lagrangian predictability). Third, the predicted attractor pattern differs from the observed one even in large scale details and in principle the model can approximate drifter trajectories only within very short time intervals (the partial Lagrangian predictability). Identification of the scenario of model Lagrangian predictability is an important task in model verification.

In the present study we a priori assume that the modeled and observed attractors are similar, i.e. at least their Lyapunov dimensions coincide. However, we can doubt that a model correctly reproduces all attractor details including vertical motions. Thus, the second scenario of Lagrangian predictability is expected to appear in our computations.

Briefly, it can be argued in the following way. Like as in a two-dimensional compressible flow the advection of floats that are not neutrally buoyant, gives rise to clustering (Falkovich et al., 2001). The floats tend to dispose along an ocean front or to reach a convergence zone. The float clustering evolution strongly depends on the structure of vertical velocity which is not in general reproduced accurately by numerical ocean models. Therefore a drift and spread of a cluster composed from the real floats differ considerably from those of a cluster of the synthetic particles even if the non-divergent velocity is modeled quite accurately.

Thus, we shouldn't expect the long-term Lagrangian predictability for a high-resolution ocean model because the real drifters and synthetic particles have different asymptotic behavior. 


\subsection{Model prediction error}

Let $P$ quasi-Lagrangian floats drogued at the vertical horizon $z$ display the spatio-temporal variability of circulation in an area of interest. The model may reproduce trajectories of synthetic floats (particles) to be compared to the real drifters.

Let the position and velocity of some drifter and synthetic particle used in the comparison be represented by

$\boldsymbol{Z}(t)=\{X(t), Y(t), U(t), V(t)\}^{t}$,

and

$Z^{\text {part }}(t)=\left\{X^{\text {part }}(t), Y^{\text {part }}(t), U^{\text {part }}(t), V^{\text {part }}(t)\right\}^{t}$,

respectively, the superscript ' $t$ ' denotes the transpose. Then, the model prediction error be represented by a 4-dimensional vector

$\delta \boldsymbol{Z}(t)=\{\delta X(t), \delta Y(t), \delta U(t), \delta V(t)\}^{t}$,

with

$\delta X(t)=X(t)-X^{\text {part }}(t), \quad \delta Y(t)=Y(t)-Y^{\text {part }}(t)$,

$\delta U(t)=U(t)-U^{\text {part }}(t), \quad \delta V(t)=V(t)-V^{\text {part }}(t)$.

Because the positioning drifter buoys by navigation systems is quite accurate with error less than $200 \mathrm{~m}$, we take that differences in initial positions of the drifter and particle equal to zero at $t_{0}$. That is not true for difference between their velocities. Thus $\delta \boldsymbol{Z}\left(t_{0}\right)=\left\{0,0, \delta U_{0}, \delta V_{0}\right\}^{t}$.

Since the MPE is a-priori stochastic, the Lagrangian predictability is effectively determined using the multi-variable PDF, $P(\delta X, \delta Y, \delta U, \delta V, t)$ and the statistical moments

$\tilde{L}_{q}=\left\langle|\delta \boldsymbol{Z}|^{q}\right\rangle, \quad q=1, \ldots, Q$.

The MPE

$J=\left\langle\left\|\delta \boldsymbol{Z}^{t} \boldsymbol{A} \delta \boldsymbol{Z}\right\|_{4}\right\rangle$,

$\|\ldots\|_{4}$ is the 4-dimensional Eucledian norm, $\boldsymbol{A}$ is the weight matrix, determines a prediction time scale $\tau$ from the condition that

$J(\tau) \leq \epsilon^{2}$,

as a time interval within which this RMS error is less than the accepted prediction accuracy $\epsilon$ (the tolerance).

In general the use of Eqs. (4) and (6) sometimes induces problems in physical interpretation of the MPE growth since different dynamical regimes of the error may correspond to the same power decay law for $\tilde{L}_{q}$ (Rangarajan and Ding, 2000). In other words, it is difficult (or even impossible) to distinguish different physical mechanisms inducing the anomalous dynamical regimes through $L_{q}$ in practical applications.

\subsection{Irreversible-skill time}

The IT $\left(\tau_{\text {irrev }}\right)$ has been proposed for measuring the prediction-skill without the Gaussian distribution assumption for MPE (Ivanov et al., 1994; Ivanov and Margolina, 1999) on the base of the first-passage time (Gardiner, 1985). This prediction time scale is determined as the time period when $J$ exceeds $\epsilon^{2}$ for the first time. The returning prediction-skill that is referred to $J$ is smaller than $\epsilon^{2}$ after IT is excluded.

In comparing with the MPE, two different statistics for IT can be defined with the initial error $\delta \boldsymbol{Z}_{0}$ and tolerance level $\epsilon$ : (a) PDF (probability density function) of IT, $W\left(\tau, \delta \boldsymbol{Z}_{0}, \epsilon\right)$, and (b) PSP (probability of successful predictions), $\left(F\left(t_{0}, \delta \boldsymbol{Z}_{0}, \epsilon, t-t_{0}\right)\right)$, which is the probability that $\tau_{\text {irrev }}$ is larger than the given time period $\left(t-t_{0}\right)$.

The PSP satisfies the so-called Pontryagin-Kolmogorov equation (Chu et al., 2002a, b, c). The statistics are connected by

$F\left(t_{0}, \delta \boldsymbol{Z}_{0}, \epsilon, t-t_{0}\right)=1-\int_{0}^{t-t_{0}} W\left(\tau, \delta \mathbf{Z}_{0}, \epsilon\right) d \tau$.

PSP is useful to verify the prediction-skill in practical applications (Ivanov et al., 1994; Ivanov and Margolina, 1999) because it is more computational feasible than the PDF of IT.

Additionally, the statistical moments of IT also estimate the practical model prediction skill. They are easily calculated by

$\tau_{k}\left(\delta \mathbf{Z}_{0}, \epsilon\right)=k \int_{t_{0}}^{\infty} F\left(t_{0}, \delta \mathbf{Z}_{0}, \epsilon, t-t_{0}\right)\left(t-t_{0}\right)^{k-1} d t$.

The mean and variance of IT are calculated as

$\left\langle\tau_{\text {irrev }}\right\rangle=\tau_{1}$,

$\left\langle\tau_{\text {irrev }}^{2}\right\rangle=2 \tau_{2}-\tau_{1}^{2}$.

The use of IT allows analytical estimating the prediciton skill through the statistical moments which are a measure of any deviations of PDF from the Gaussian shape (Ivanov et al., 1994; Chu et al., 2002a, b, c). Besides, robust algorithms for the estiamtion of PDF and PSP from numerical modeling are developed by Ivanov et al. (1999) and Chu et al. (2002a, 2003c) even when the prediciton skill is controlled by several variables of model input.

\subsection{Singular vector approach}

This approach, commonly used to estimate large atmospheric model prediction-skill, is valid only for small MPEs (Palmer, 2000). The MPE dynamics is the linearization with respect to the reference trajectory $\boldsymbol{Z}_{\text {ref }}$ described by the following tangent-linear model (Lorenz, 1965; Lacarra and Talagrand, 1988 and others) within the reference period $\left(T-t_{0}\right)$.

$$
\begin{aligned}
& \frac{d}{d t} \delta \boldsymbol{Z}(t)=\boldsymbol{J}_{1}\left[\boldsymbol{Z}_{\mathrm{ref}}(t)\right] \delta \boldsymbol{Z}, \\
& \delta \boldsymbol{Z}\left(t_{0}\right)=O\left[\boldsymbol{Z}_{\mathrm{ref}}\left(t_{0}\right)\right] \quad t \subset\left[t_{0}, T\right],
\end{aligned}
$$


where

$\left(\boldsymbol{J}_{1}\right)_{i j}=\frac{\partial H_{i}\left(\boldsymbol{Z}_{\text {ref }}\right)}{\partial x_{j}}$

is the Jacoby matrix; $H$ is the dynamical operator of a prediction model.

The formal solution of tangent-linear Eq. (11) is given by

$\delta \boldsymbol{Z}(t)=\boldsymbol{G}\left(t, t_{0}\right) \delta \boldsymbol{Z}\left(t_{0}\right)$,

where $\boldsymbol{G}\left(t, t_{0}\right)$ is the transition operator.

The eigenvalues and eigenfunctions of the operator

$\boldsymbol{L}=\boldsymbol{G}^{t} \boldsymbol{G}$

are called the singular values and vectors ("the optimal perturbations"), respectively. The directions, along which the growth of $\delta \boldsymbol{Z}\left(t_{0}\right)$ to $\delta \boldsymbol{Z}(t)$ is most fast, are identified by the singular vectors. This growth leads to the maximization of the amplification factor

$F=\frac{(\boldsymbol{G} \cdot \delta \boldsymbol{Z}, \boldsymbol{G} \cdot \delta \boldsymbol{Z})}{(\delta \boldsymbol{Z}, \delta \boldsymbol{Z})}=\frac{(\boldsymbol{L} \cdot \delta \boldsymbol{Z}, \delta \boldsymbol{Z})}{(\delta \boldsymbol{Z}, \delta \boldsymbol{Z})}$.

Geometrically the equation

$(\boldsymbol{L} \cdot \delta \boldsymbol{Z}, \delta \boldsymbol{Z})=1$,

defines a hyper-ellipsoid (ellipsoid of prediction uncertainty) with the smallest axis maximizing the amplification factor $F$. This axis is associated with the largest singular value. The fastest growing perturbations are the singular vectors corresponding to the largest singular values. Knowledge of the leading singular vectors allows computating the amplification factor and $e$-folding predictability time (Palmer, 2000).

The singular vectors and values have not been yet applied as a measure of Lagrangian model predictability, before. Thus, two problems will be in focus of the present study. First, we would like to develop a simple approach to compute the singular values and vectors. Second, we need to estimate the reference period from the real oceanographic observations.

\subsection{Ensemble realizations}

It was pointed out in numerous studies (for further discussions see Palmer, 2000) that error growth may, in fact, be highly dependent on the local properties along the attractor. Thus our goal is to determine "the global" mean prediction skill (where "global" average is to be understood as a mean over the circulation attractor). Another reason to average along the attractor is because in oceanographic practice the numbers of drifters and comparative events when the drifter and particle positions are compared, are not large. For example, only 55 drifters moved in the Gulf of Mexico within the time period under investigation. Therefore, we need to construct an ensemble for statistical significant estimations from such poor observations.

Ideally, the averaging may be constructed in the following way. Utilizing observations from $P$ Lagrangian drifters we firstly reconstruct the spatial structure of circulation in the area of interest and compare it with computed by a model in a prediction metric. For simplicity let us take the traditional RMS error written as

$$
\begin{aligned}
& \left\langle\left\langle\delta \boldsymbol{Z}^{2}\left(t, \boldsymbol{Z}_{\text {ref }}\left(t_{0}\right)\right)\right)\right\rangle_{\text {theor }}= \\
& \int \delta \boldsymbol{Z}^{2}(t) f_{1}\left(\delta \boldsymbol{Z}, \boldsymbol{Z}_{\text {ref }}\left(t_{0}\right), t\right) d \delta \boldsymbol{Z},
\end{aligned}
$$

where $f_{1}$ is the PDF for a model prediction error over the area of interest. We specially stressed in Eq. (14) that the model prediction skill in general must depend on initial position $\boldsymbol{Z}_{\text {ref }}\left(t_{0}\right)$ along the attractor trajectory.

The mean error is determined through averaging Eq. (14) over the attractor trajectory

$$
\begin{aligned}
& \left\langle\delta \boldsymbol{Z}^{2}(t)\right\rangle_{\text {theor }}= \\
& \quad \int\left\langle\left\langle\delta \boldsymbol{Z}^{2}\left[t, \boldsymbol{Z}_{\text {ref }}\left(t_{0}\right)\right]\right\rangle\right\rangle_{\text {theor }} B\left[\boldsymbol{Z}_{\text {ref }}\left(t_{0}\right)\right] f_{2}\left[\boldsymbol{Z}_{\text {ref }}\left(t_{0}\right)\right] d \boldsymbol{Z}_{\text {ref }}
\end{aligned}
$$

where $f_{2}$ describes the distribution of comparative events along the attractor, $B$ is the weight accounting the heterogeneous feature of the attractor.

In practice Eq. (14) is transformed to

$$
\left\langle\left\langle\delta \boldsymbol{Z}^{2}(t)\right\rangle\right\rangle=\frac{1}{P} \sum_{p=1}^{P} \delta \boldsymbol{Z}_{p}^{2}\left[t, \boldsymbol{Z}_{\mathrm{ref}}^{p}\left(t_{0}\right)\right]
$$

It was pointed out in Vapnik (1983) and Chu et al. (2003a, b) that comparative events may be selected so that for the empirical average $\left\langle\left\langle\delta \boldsymbol{Z}^{2}(t)\right\rangle\right\rangle$ with the probability larger than $\chi$, homogeneously (without excurses) tends to the theoretical one if $P \rightarrow \infty$, i.e.

$$
\begin{gathered}
\operatorname{Prob}\left\{\sup \mid\left\langle\left\langle\delta \boldsymbol{Z}^{2}\left[t, \boldsymbol{Z}_{\text {ref }}\left(t_{0}\right)\right]\right\rangle\right\rangle_{\text {theor }}\right. \\
\left.-\left\langle\left\langle\delta \boldsymbol{Z}^{2}(t)\right\rangle\right\rangle \mid>\chi\right\} \rightarrow 0
\end{gathered}
$$

This explains why in practice impact of the spatial heterogeneity on the estimations of model prediction skill may be strongly reduced even for quite heterogeneous drifter covering an area of interest.

The most dangerous procedure is the averaging over an attractor trajectory. In the present study we will apply the following procedure

$$
\left\langle\delta \boldsymbol{Z}^{2}(t)\right\rangle=\int f_{2}\left(\boldsymbol{Z}_{\mathrm{ref}}\right)\left\langle\left\langle\delta \boldsymbol{Z}^{2}\left[t, \boldsymbol{Z}_{\mathrm{ref}}\left(t_{0}\right)\right]\right\rangle\right\rangle d \boldsymbol{Z}_{\mathrm{ref}}
$$

where $B=1$.

Operator (18) represents a two-step averaging process: (a) over each pair of drifter-particle and (b) along the attractor trajectory. In the present study, $f_{2}$ is assumed to be the continuous uniform distribution function, i.e. the model prediction skill is stationary or at least very slowly variable within the reference period.

This is a quite strong hypothesis and may not be relaxed here, because the number of drifter buoys equals only to 55 . 


\section{Structure of model attractor}

\subsection{Model circulation}

To show the verification of the Lagrangian predictability as an example the Gulf of Mexico real time nowcast/forecast system is taken. This system (Kantha et al., 1999; Schaudt et al., 2001; see also Kantha and Clayson, 2000) is based on the University of Colorado version of the Princeton Ocean Model (POM) with $1 / 12^{\circ}$ horizontal resolution and $24 \sigma$-levels. The water inflow through the Yukatan Channel has been prescribed, the outflow through the Strait of Florida evolved with the model physics. The model reaches quasi steady regimes after 10 year integration. High-resolution bathymetry (Fig. 1a), the real-time altimetric sea surface height (SSH) anomalies derived from NASA/CNES TOPEX and ESA ERS-1/2 altimeters, and composite MCSST data derived from NOAA AVHRR assimilated into the model in a continuous data assimilation mode are used to produce a nowcast and a four-week forecast. Kantha and Clayson (2000) found that forecast retains considerable skill to about 1-2 weeks, beyond which the forecast begins to deviate from reality.

Since the Lagrangian drifters are drogued at $50 \mathrm{~m}$ depth, the modeled horizontal velocity field at the same depth is used for the model-data comparison in 1998, throughout this paper. The model successfully reproduces the typical circulation patterns (the Loop Current and eddy shedding) in the Gulf of Mexico (Fig. 1b,c). The anti-cyclonic eddies are regularly pinched off from the Loop Current, which enters the eastern Gulf of Mexico through the Yucatan Channel and exits through the Strait of Florida. The Loop Current meanders and eddies (most distinguished mesoscale features) are predicted with radii less than $100 \mathrm{~km}$ and velocities larger than $100 \mathrm{~cm} / \mathrm{s}$.

\subsection{Toroidal and poloidal EOF decomposition}

The model circulation at $50 \mathrm{~m}$ depth was analyzed as a 2D divergent flow. Helmholtz (Morse and Feshbach, 1953) or Moffat-Zeldovich (Moffat, 1978; Zeldovich et al., 1985) decomposition may be applied in this case. Our study uses the MZD to construct appropriate EOFs for the analysis of the spatio-temporal structure of the model attractor at $50 \mathrm{~m}$ depth. Other oceanographic applications of MZD can be found in Eremeev et al. (1992), Lipphardt et al. (2000) and Chu et al. (2003a, b) selected as examples.

Let us briefly explain the MZD. It represents 3-D velocity $\left\{\boldsymbol{u}_{\perp}, w\right\}$ at any vertical horizon $\tilde{z}=$ const as

$\boldsymbol{u}_{\perp}=\boldsymbol{u}^{(T)}+\boldsymbol{u}^{(P)}$,

$\boldsymbol{u}^{(T)}=\nabla \times(\boldsymbol{k} \Psi), \quad \boldsymbol{u}^{(P)}=\nabla \times \nabla \times(\boldsymbol{k} \Phi)$,

$w=-\nabla \cdot \nabla \Phi$,

where $\boldsymbol{k}$ is the unit vector perpendicular to the surface $\tilde{z}=$ const; $\boldsymbol{u}_{\perp}=(u, v)$ and $w$ are the horizontal and vertical velocity components, respectively; $\nabla=\left(\nabla_{x}, \nabla_{y}\right)$ is the gradient operator. The $\Psi$ (toroidal) and $\Phi$ (poloidal) potentials
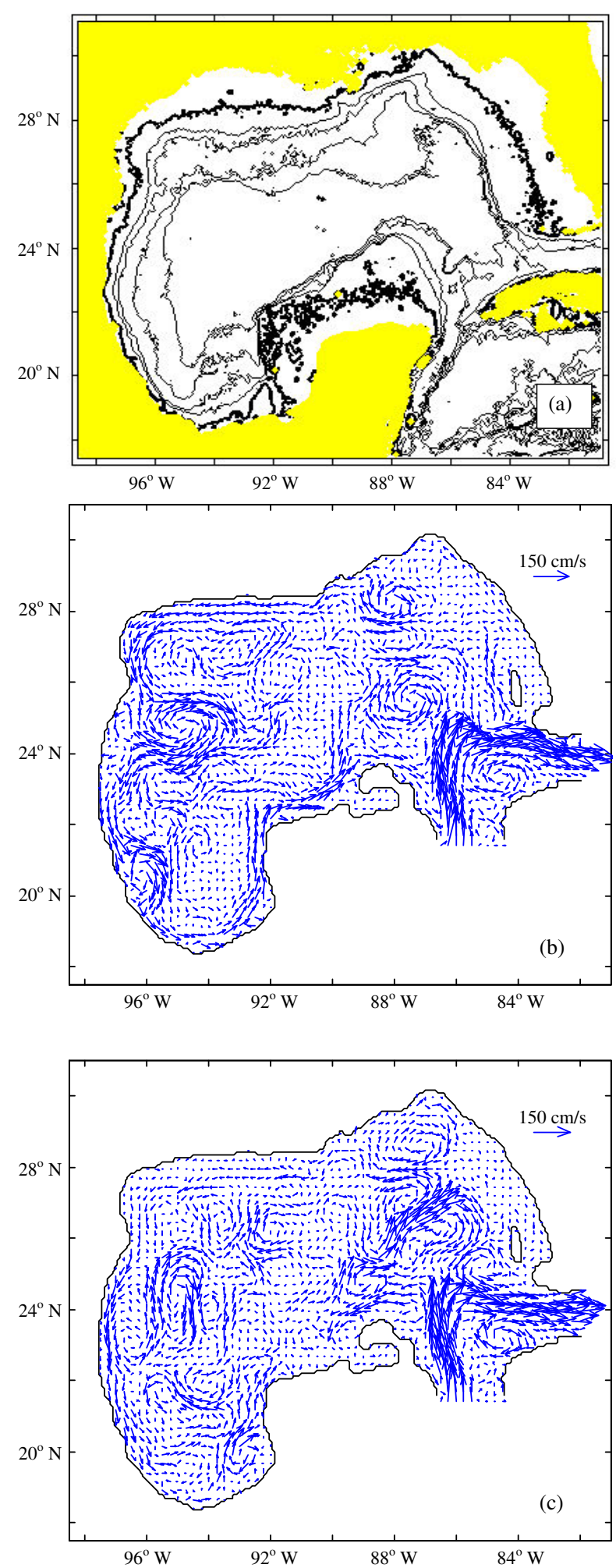

Fig. 1. High-resolution bathymetry utilized by the model (a) and circulation patterns in the Gulf of Mexico for two different dynamical regimes in 1998 on (b) Julian Day 260, and (c) Julian Day 320. The bold line in (a) is $50 \mathrm{~m}$ isobath. Transition from one regime to another is characterized by energy cascade from low- to high-order EOFs. 
generate the toroidal $\left(\boldsymbol{u}^{(T)}\right)$ and poloidal $\left(\boldsymbol{u}^{(P)}\right)$ velocities, respectively.

Knowledge of the potentials $\Psi$ and $\Phi$ allows computing EOFs separately for each of these potentials: $\Psi_{n}(x, y, \tilde{z})$, $n=1, \ldots, N$ (toroidal modes) and $\Phi_{m}(x, y, \tilde{z}), m=1, \ldots, M$ (poloidal modes).

The following scheme was chosen for practical computations of the EOFs. Let $T-t_{0}$ be the reference period during which the model-data comparison takes place. In our case this time period can be chosen as not larger than 1 year. Then, the horizontal velocity $\boldsymbol{u}_{\perp}=\left(\boldsymbol{u}^{(T)}, \boldsymbol{u}^{(P)}\right)$ and the potentials $(\Psi, \Phi)$ were represented as combinations of stationary within the reference period currents (SPCs) $\overline{\boldsymbol{u}}_{\perp}=\left(\overline{\boldsymbol{u}}^{(T)}, \overline{\boldsymbol{u}}^{(P)}\right), \bar{\Psi}, \bar{\Phi}$ and pulsation components $\boldsymbol{u}_{\perp}^{\prime}=\left(\boldsymbol{u}^{(T)}, \boldsymbol{u}^{(P)}\right), \Psi^{\prime}, \Phi^{\prime}$ :

$\Psi(x, y, \tilde{z}, t)=\bar{\Psi}(x, y, \tilde{z})+\Psi^{\prime}(x, y, \tilde{z}, t)$,

$\boldsymbol{u}_{\perp}^{(P)}=\overline{\boldsymbol{u}}^{(P)}+\boldsymbol{u}^{(P)}$,

$\Phi(x, y, \tilde{z}, t)=\bar{\Phi}(x, y, \tilde{z})+\Phi^{\prime}(x, y, \tilde{z}, t)$,

$\boldsymbol{u}_{\perp}^{(T)}=\overline{\boldsymbol{u}}^{(T)}+\boldsymbol{u}^{\prime(T)}$.

Two sets of EOFs which are the eigenfunctions of a large $18200 \times 18200$ matrix, were computed for $\Psi^{\prime}$ and $\Phi^{\prime}$ through the algorithm of Penenko and Protasov (1978). To compute EOFs we used the same grid as in the hydrodynamic model and circulation velocity through each two days of model calculations. Numerical experiments demonstrated that for the reference period larger than 8 months the computed EOFs were practically not sensitive to increasing length of this period.

The spatial inhomogeneity and temporal intermittency of the circulation attractor are analyzed through the spatial structure of EOFs and behavior of mode amplitudes $A_{n}$ and $B_{m}$ calculated by the inner products $A_{n}=\left(\Psi^{\prime} \cdot \Psi_{n}\right)$ and $B_{m}=\left(\Phi^{\prime} \cdot \Phi_{m}\right)$, respectively.

\subsection{Spatial structure of model attractor}

\subsubsection{Stationary part}

Both the toroidal $\left(\overline{\boldsymbol{u}}^{(T)}\right)$ (Fig. 2a) and poloidal $\left(\overline{\boldsymbol{u}}^{(P)}\right)$ (Fig. 2b) SPCs have simple large-scale topology. The toroidal circulation explicitly dominates over the poloidal one. This indicates the quasi-geostrophic nature of SPC patterns. A measure of toroidal circulation impact is the compressibility factor (Schumacher and Eckartdt, 2002)

$\sigma=\frac{\left\langle\left\langle\left(\nabla \cdot \boldsymbol{u}_{\perp}\right)^{2}\right\rangle\right\rangle}{\left\langle\left\langle\left(\nabla \boldsymbol{u}_{\perp}\right)^{2}\right\rangle\right.}$,

i.e. the ratio of the mean square divergence versus the mean square velocity gradient. Here, the double brackets, also as in Eq. (14), denote averaging over an area of interest. The compressibility factor of the model SPC is about 0.05 . However, the poloidal SPC $\left(\overline{\boldsymbol{u}}^{(P)}\right)$ (Fig. 2b) clearly shows the existence of the convergence and divergence zones in the circulation patterns.
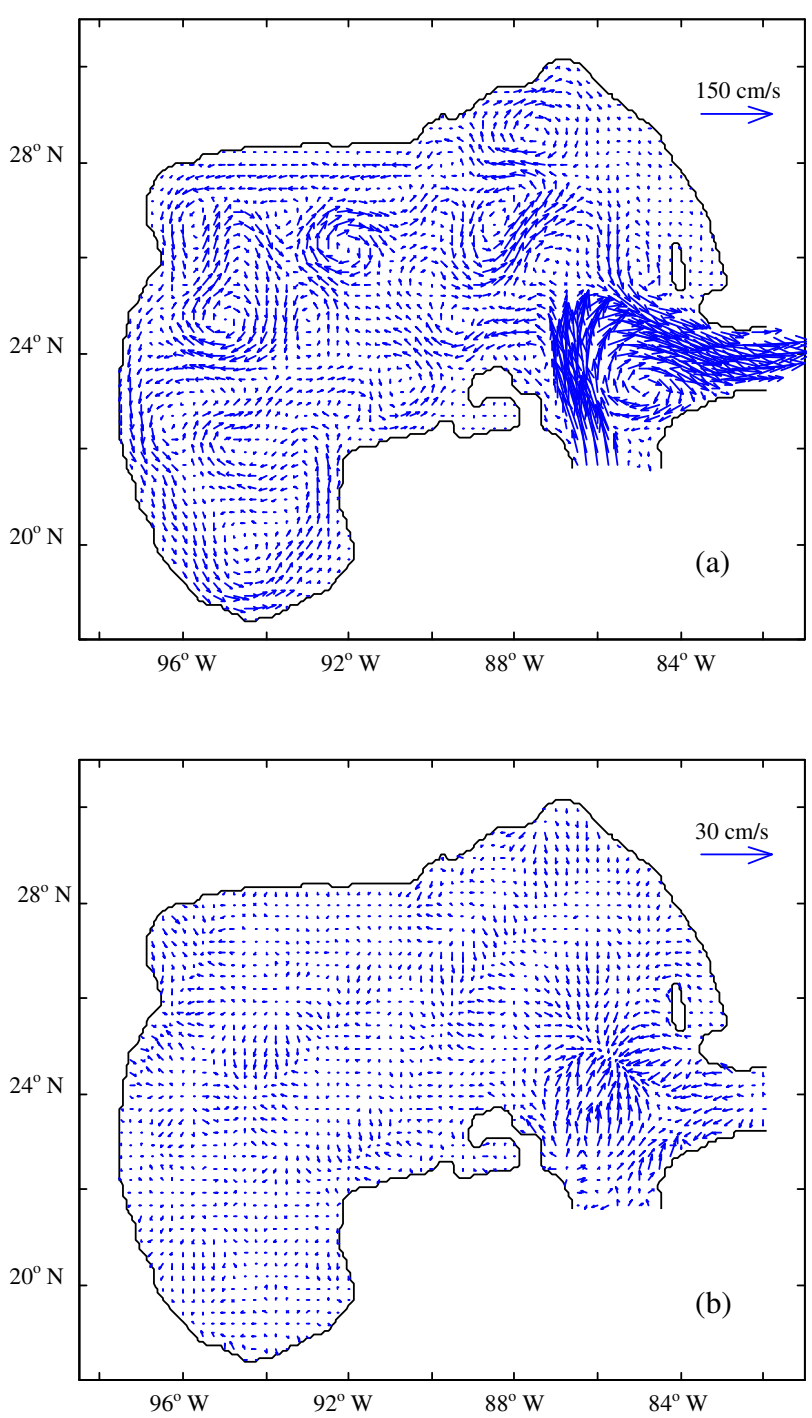

Fig. 2. Structures of the toroidal (a) and poloidal (b) SPCs.

\subsubsection{Pulsation component of circulation}

The spatial structures of the non-stationary part are represented by various toroidal $(n=1, \ldots, N)$ and poloidal $(m=1, \ldots, M)$ velocity modes (snapshots) $\left\{\boldsymbol{u}_{n}^{(T)}=\nabla \times\left[\boldsymbol{k} \Psi_{n}\right]\right\}$ and $\left\{\boldsymbol{u}_{m}^{(P)}=\nabla \cdot \boldsymbol{k} \Phi_{m}\right\}$. The most striking features of $\left\{\boldsymbol{u}_{n}^{(T)}\right\}$ are (a) the multi-eddy structures with smaller eddy scales for high-order modes, (b) the Loop Current occurring only in the first toroidal mode $\boldsymbol{u}_{1}^{(T)}$ but not in other modes and (c) small-scale eddies contributing in the low-order modes as well. For example, the first toroidal velocity mode $\boldsymbol{u}_{1}^{(T)}$ represents a combination of the Loop Current and multi-eddy structures (Fig. 3a). The second toroidal velocity mode $\boldsymbol{u}_{2}^{(T)}$ (Fig. 3b) shows the existence of multi-eddy structures such as dipoles. The most striking feature of $\left\{\boldsymbol{u}_{m}^{(P)}\right\}$ is that the convergence and divergence zones at the mesoscales in the deep basin are represented only by the poloidal velocity modes with order higher than 

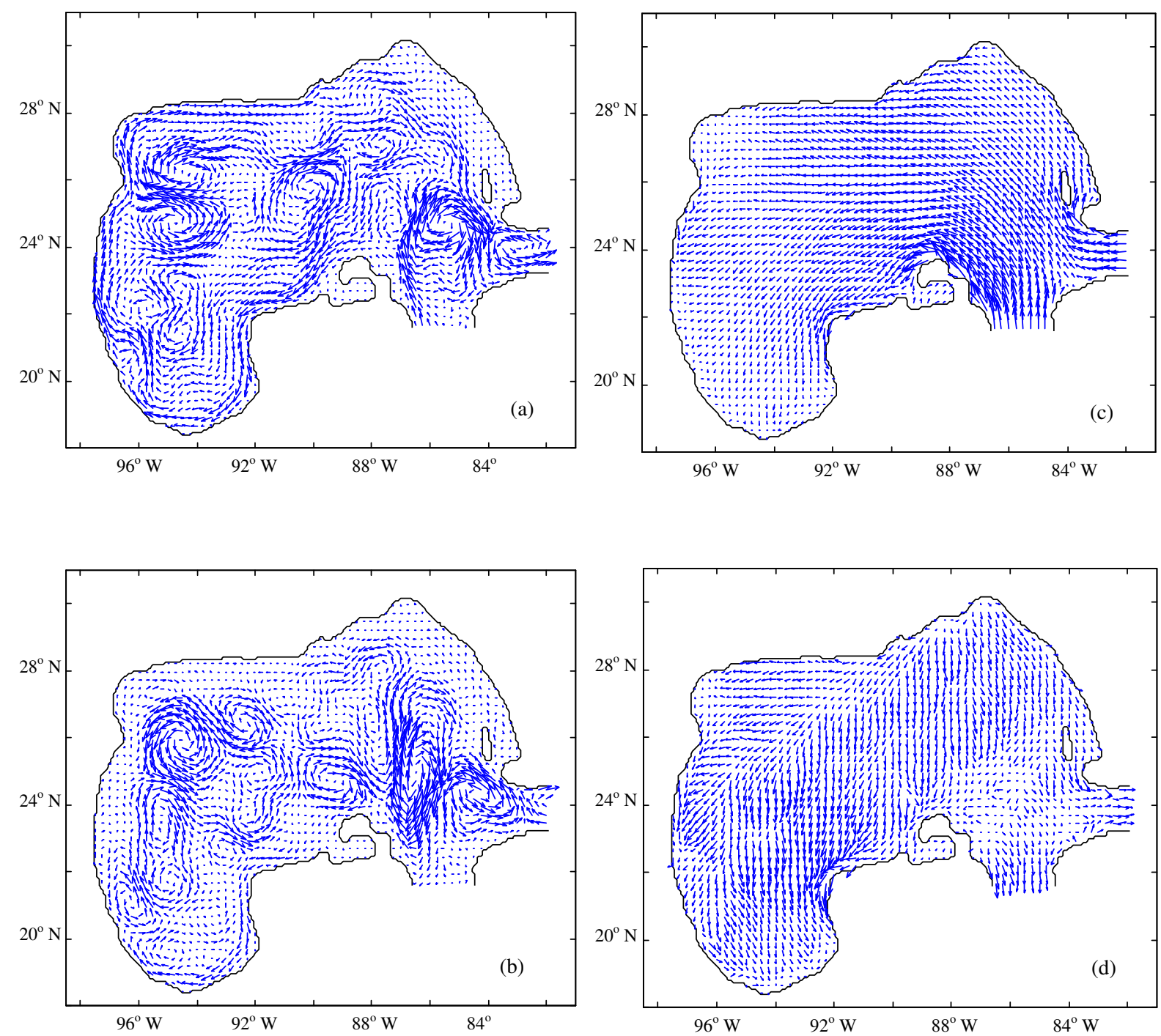

Fig. 3. Low-order toroidal $[\mathrm{n}=1(\mathbf{a}), \mathrm{n}=2(\mathbf{b})]$ and poloidal $[\mathrm{m}=1(\mathbf{c}), \mathrm{m}=2(\mathbf{d})]$ EOFs.

two. The first $\left(\boldsymbol{u}_{1}^{(P)}\right)$ (Fig. 3c) and the second $\left(\boldsymbol{u}_{2}^{(P)}\right)$ (Fig. 3d) poloidal velocity modes display the large-scale motions as well as the upwelling and downwelling in the coastal zone of the Gulf of Mexico.

The fractions of energy for each type of EOFs grow rapidly with the increase of the mode numbers $(N, M)$ (Fig. 4a, b). Here, more than $90 \%$ of the toroidal and $99 \%$ of the poloidal kinetic energy lie in the first ten toroidal and two poloidal modes, respectively. The first toroidal mode contains more than $45 \%$ of the total energy of toroidal motions.

\subsection{Temporal structure of model attractor}

Temporal variations of the first ten toroidal mode amplitudes $\left\{A_{1}(t), \ldots A_{10}(t)\right\}$ (Fig. 5) show various degrees of complexity, which increases in the higher-order modes. Fairly large excursions from the mean are also noted. The temporal variability scale of the second (Fig. 5b) and third (Fig. 5c) modes exceeds the temporal variability scale for the first mode (Fig. 5a). Amplitudes of the sixth (Fig. 5f), ninth (Fig. 5i), and tenth (Fig. 5j) modes demonstrate irregular behaviors. Temporal variations of the first two poloidalmode amplitudes $\left\{B_{1}(t), B_{2}(t)\right\}$ show the similar complexity (Figs. 5k and 51).

The pulsations of mean toroidal $\left\langle\left\langle E_{\mathrm{tor}}^{\prime}\right\rangle\right)$ and poloidal $\left\langle\left\langle E_{\mathrm{pol}}^{\prime}\right\rangle\right\rangle$ energies also demonstrate complex irregular (abrupt) behavior (Fig. 6a, b). The $\left\langle\left\langle E_{\text {tor }}^{\prime}\right\rangle\right\rangle$ varies between 240 and $600 \mathrm{~cm}^{2} / \mathrm{s}^{2}$ with energy peeks occurring at Julian Days-150, $-210,-300$, and -340 of 1998 (Fig. 6a).The relative variations of $\left\langle\left\langle E_{\mathrm{pol}}^{\prime}\right\rangle\right\rangle$ are much stronger, between 10 and $80 \mathrm{~cm}^{2} / \mathrm{s}^{2}$, with explicit energy peeks at Julian Day-230, -245 and -360 of 1998 (Fig. 6b). The behavior of $\left\langle\left\langle E_{\text {tor }}^{\prime}\right\rangle\right.$ is de-locked with the variations of $\left\langle\left\langle E_{\mathrm{pol}}^{\prime}\right\rangle\right\rangle$. 

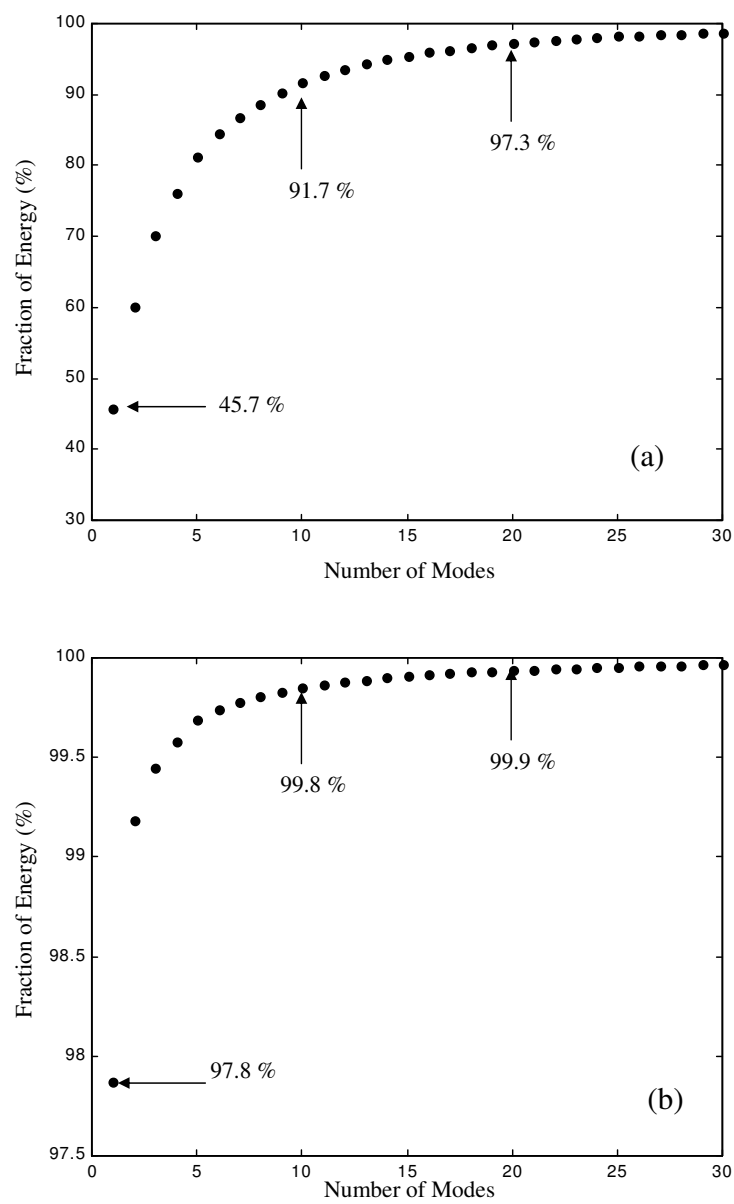

Fig. 4. The fractions of toroidal (a) and poloidal (b) energies. Arrows indicate the fraction of energy corresponding to 1,10 and 20 EOF decomposition.

The complexity in the circulation attractor behavior can be also analyzed using the four non-dimensional ratios:

$\eta_{1}=\frac{\left\langle\left\langle E_{\mathrm{pol}}\right\rangle\right\rangle}{\left\langle\left\langle E_{\mathrm{tor}}\right\rangle\right\rangle}, \eta_{2}=\frac{\left\langle\left\langle E_{\mathrm{pol}}^{\prime}\right\rangle\right\rangle}{\left\langle\left\langle E_{\mathrm{tor}}^{\prime}\right\rangle\right\rangle}, \eta_{3}=\frac{\left\langle\left\langle\bar{E}_{\mathrm{tor}}\right\rangle\right\rangle}{\left\langle\left\langle E_{\mathrm{tor}}\right\rangle\right\rangle}, \eta_{4}=\frac{\left\langle\left\langle\bar{E}_{\mathrm{pol}}\right\rangle\right\rangle}{\left\langle\left\langle E_{\mathrm{pol}}\right\rangle\right\rangle}$, and the global entropy (Aubry et al., 1991). Here, the mean total $\langle\langle E\rangle\rangle$, toroidal $\left\langle\left\langle E_{\mathrm{tor}}\right\rangle\right\rangle$ and poloidal $\left\langle\left\langle E_{\mathrm{pol}}\right\rangle\right\rangle \mathrm{ki}-$ netic energies are computed as $\langle\langle E\rangle\rangle=\left\langle\left\langle E_{\text {tor }}\right\rangle\right\rangle+\left\langle\left\langle E_{\text {pol }}\right\rangle\right\rangle$, $\left\langle\left\langle E_{\text {tor }}\right\rangle\right\rangle=\left\langle\left\langle\bar{E}_{\text {tor }}\right\rangle\right\rangle+\left\langle\left\langle E_{\text {tor }}^{\prime}\right\rangle\right\rangle$ and $\left\langle\left\langle E_{\text {pol }}\right\rangle\right\rangle=\left\langle\left\langle\bar{E}_{\text {pol }}\right\rangle\right\rangle+\left\langle\left\langle E_{\text {pol }}^{\prime}\right\rangle\right\rangle$, respectively.

The ratios $\eta_{1}$ and $\eta_{2}$ vary between $2 \%$ and $12 \%$ (Fig. 7a) and between $2 \%$ and $21 \%$ (Fig. $7 \mathrm{~b}$ ), respectively. The behavior of both the ratios are locked. That indicates the same forcing mechanism for the Gulf of Mexico circulation at the large and meso-scales.

The ratios $\eta_{3}$ and $\eta_{4}$ fluctuate from $42 \%$ to $73 \%$ (Fig. 7c) and from $3 \%$ to $27 \%$ (Fig. 7d), respectively. We see that the pulsations of the poloidal component contribute very strong into the total poloidal circulation. That stresses our conclusion about impossibility of the global Lagrangian predictability for high-resolution regional models.
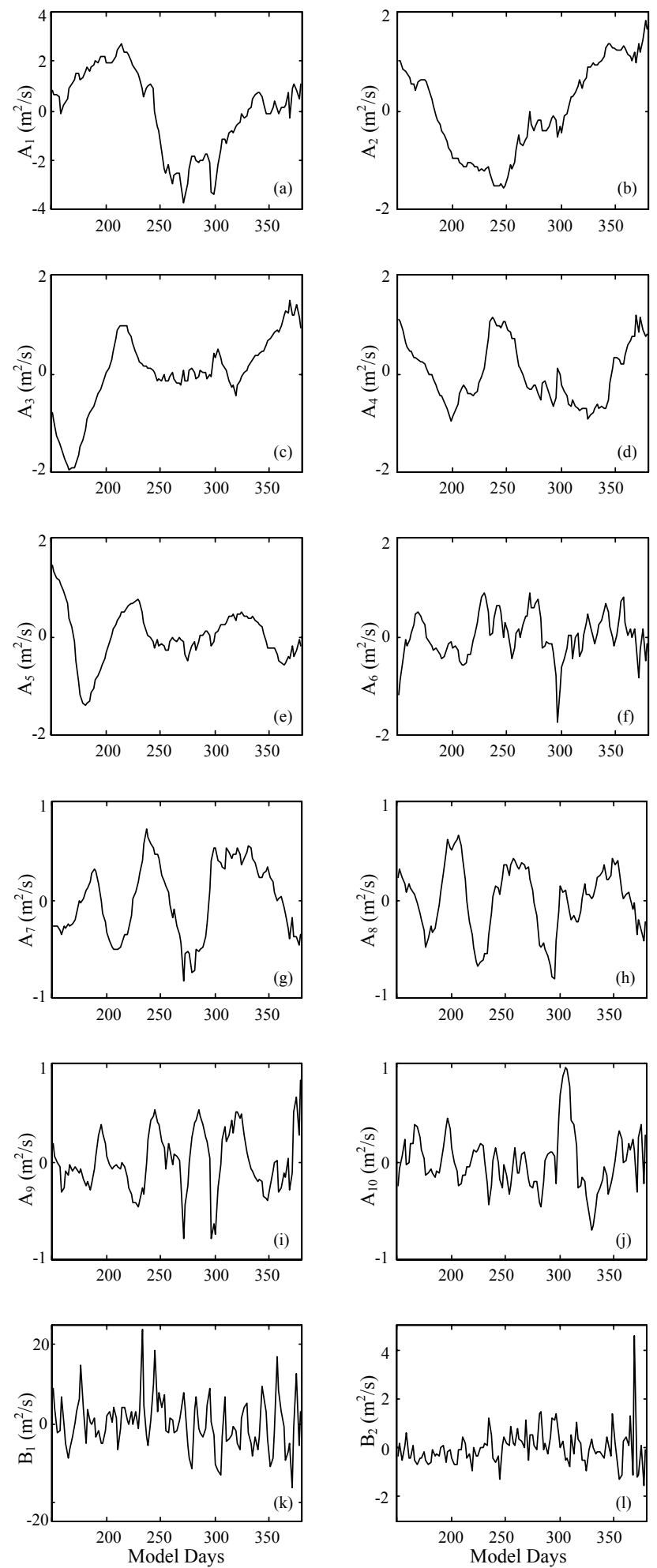

Fig. 5. Temporal evolution of mode amplitudes $A_{1}, \ldots, A_{10}$ at (a), ., (j) and $B_{1}, B_{2}$ at (k), (l). 

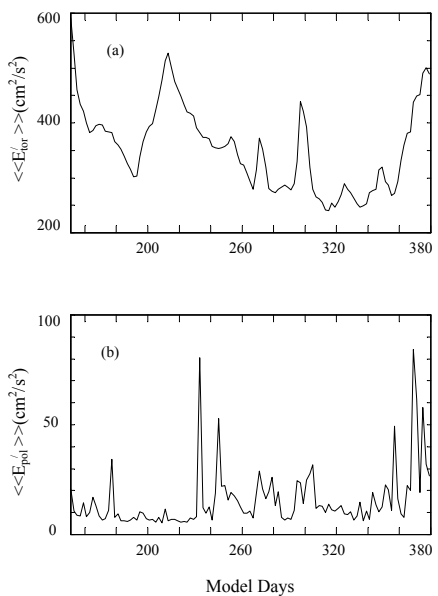

Fig. 6. Temporal evolution of $\left\langle\left\langle E_{\text {tor }}^{\prime}\right\rangle\right\rangle$ (a) and $\left\langle\left\langle E_{\text {pol }}^{\prime}\right\rangle\right\rangle$ (b).
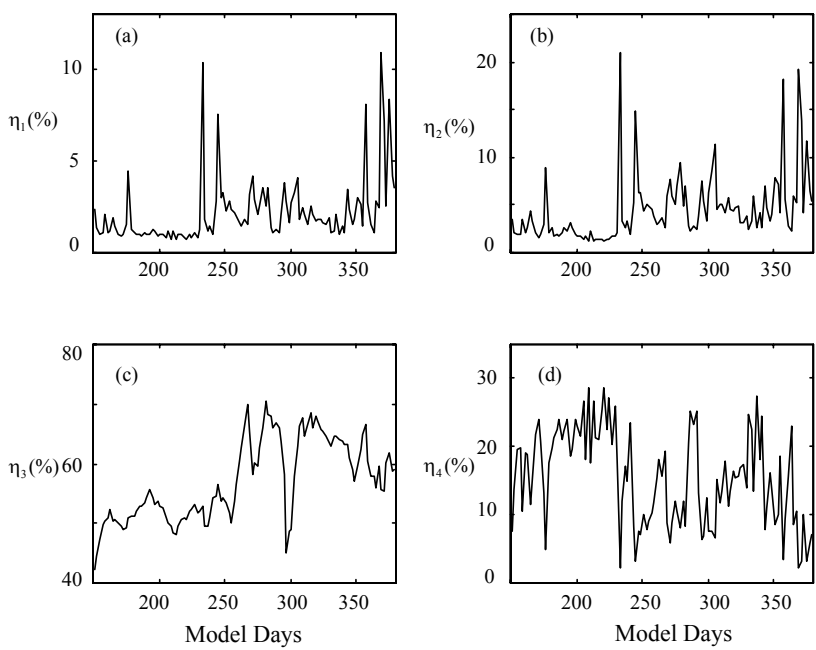

Fig. 7. Temporal evolution of non-dimensional ratios $\eta_{1}$ (a), $\eta_{2}$ (b), $\eta_{3}(\mathbf{c})$ and $\eta_{4}(\mathbf{d})$.

Two global entropies, toroidal $\left(S_{\text {tor }}\right)$ and poloidal $\left(S_{\mathrm{pol}}\right)$ can be calculated for the toroidal and poloidal movements, respectively, as

$S_{\mathrm{tor}}=-\sum_{n=1}^{N} p_{n} \log p_{n} / \log N, \quad p_{n}=A_{n}^{2} / \sum_{n=1}^{N} A_{n}^{2}$,
$S_{\mathrm{pol}}=-\sum_{m=1}^{M} p_{m}^{*} \log p_{m}^{*} / \log M, \quad p_{m}^{*}=B_{m}^{2} / \sum_{m=1}^{M} B_{m}^{2}$

The entropy $S_{\text {tor }}$ or $S_{\text {pol }}$ is 0 (total order) if and only if the first EOF mode dominates. If the energy is distributed among the modes equally (total disorder), the entropy takes its maximum value, namely 1 . Thus, the global entropies are a measure of degree of order or disorder in behavior of a model attractor.

The global toroidal and poloidal entropies with mode truncation, $N=M=16$, and $N=M=50$, are illustrated in Fig. 8 . Both these entropies display intermittent characteristics by
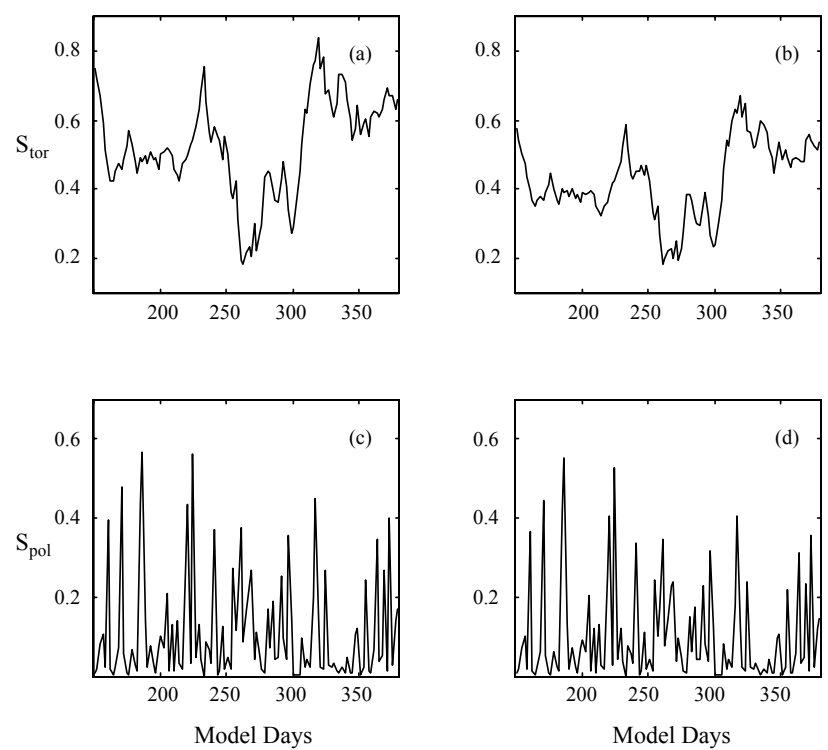

Fig. 8. Temporal behavior of the toroidal $(\mathbf{a}, \mathbf{b})$ and poloidal $(\mathbf{c}, \mathbf{d})$ entropies computed for $16(\mathrm{a}, \mathrm{c})$ and $50(\mathrm{~b}, \mathrm{~d})$ EOFs.

successive redistribution of energy initially concentrated in a few first low-order modes ( $S_{\text {tor }}=0.2$ on Julian Day-260), between other low- and high-order EOFs. Such intermittency is more evident in the poloidal field (Fig. 8c, d) than in the toroidal one (Fig. 8a, b). As a result displayed in Fig. 9a-d is the existence of irregular (probably, stochastic) pathways of divergence and convergence zones.

\section{Lyapunov dimension of model attractor}

The global prediction feature of the model attractor can be identified through different ergodic measures, such as Lyapunov dimension ( $\left.D^{\text {Lyap }}\right)$, Kolmogorov-Sinai entropy and others (Zaslavsky, 2002). Exact determination of them for a high-resolution oceanographic model is a very hard task. However, coarse estimations of $D^{\text {Lyap }}$ are possible through the approach originally developed by Syrovich (1989).

The minimum mode truncation number required for accurate representing the circulation as measured by the energy norm is treated as the intrinsic dimension $D^{\text {int }}$ of phase space generated by the basis $\left\{\Psi_{n}(x, y)\right\}$ and $\left\{\Phi_{m}(x, y)\right\}$. This dimension is identified as the number of modes requested so that the captured energy is at least $90 \%$ of the total one and so that no neglected mode, on average, contains more than $1 \%$ of the energy lying in the most energy mode (Syrovich, 1989).

It was obtained in numerous studies, here we cite only Syrovich (1989) and Aubry et al. (1991), that the intrinsic and Lyapunov dimensions are connected through the following empirical relation

$\left[D^{\text {Lyap }}\right]=D^{\text {int }}-1$,

where $\left[D^{\text {Lyap }}\right]$ is the integer part of $D^{\text {Lyap }}$. 


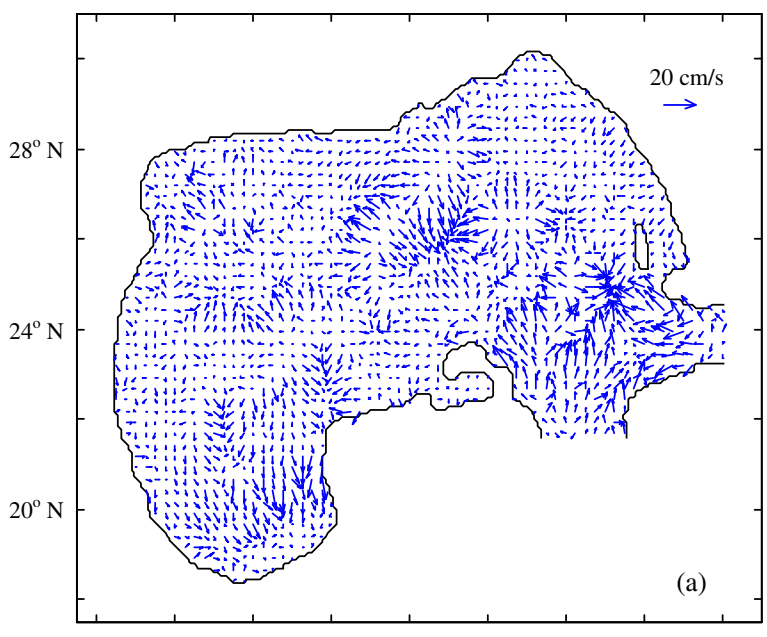

$96^{\circ} \mathrm{W}$

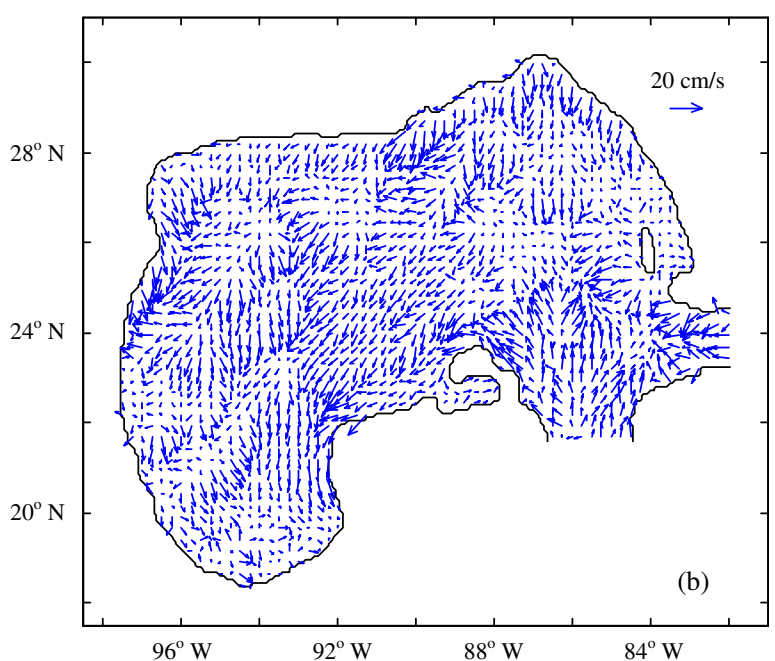

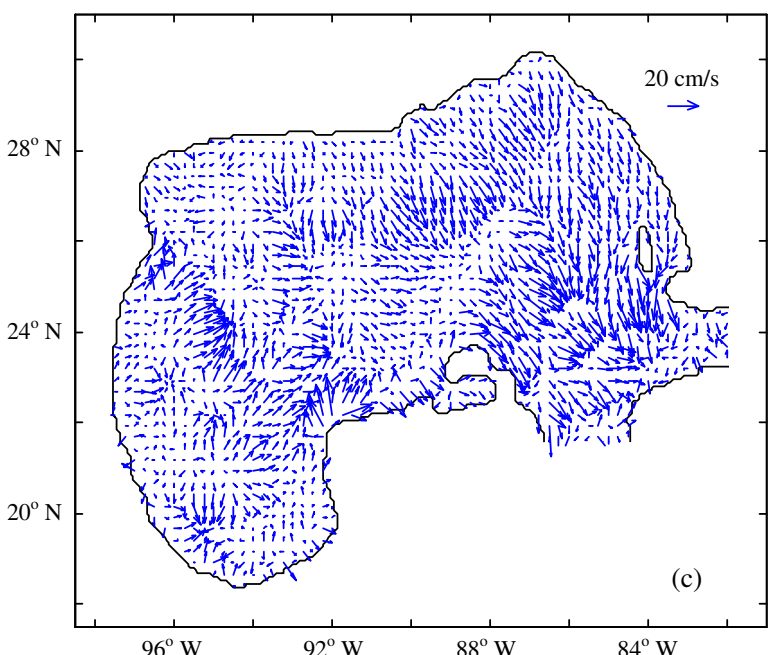

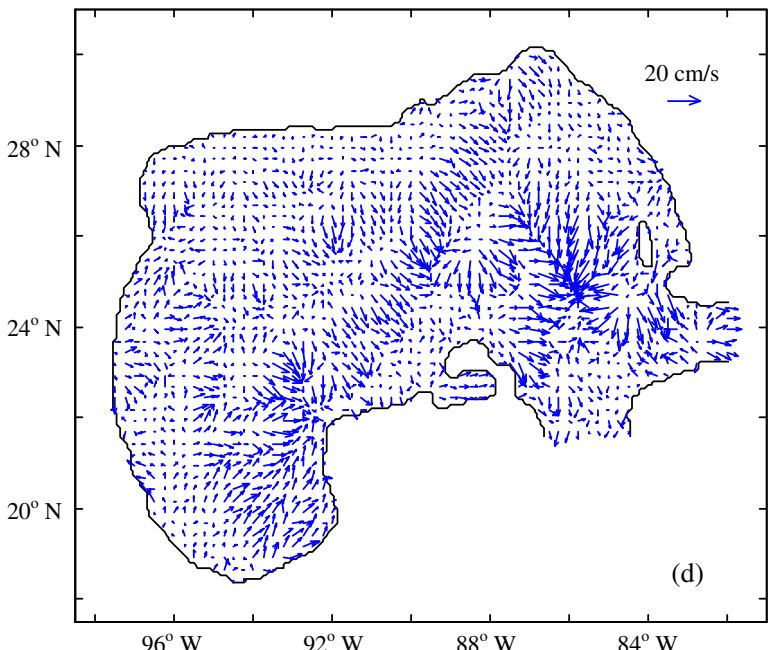

Fig. 9. Model reproduced poloidal circulation in 1998 on Julian Day 200 (a), Julian Day 235 (b), Julian Day 300 (c), and Julian Day 360 (d).

Since the circulation is decomposed into the toroidal and poloidal parts, two different intrinsic dimensions and naturally two Lyapunov dimensions should be introduced as $\left(D_{\mathrm{tor}}^{\text {int }}, D_{\text {tor }}^{\text {Lyap }}\right)$ and $\left(D_{\text {pol }}^{\text {int }}, D_{\text {pol }}^{\text {Lyap }}\right)$ for the toroidal and poloidal velocity components, respectively. The analysis of fractions of toroidal and poloidal energies (see Fig. 4a, b) results in

$$
\begin{array}{ll}
D_{\text {tor }}^{\text {int }} \sim 16 ; & D_{\text {pol }}^{\text {int }} \sim 2 ; \\
15 \leq D_{\text {tor }}^{\text {Lyap }} \leq 16 & 1 \leq D_{\text {pol }}^{\text {Lyap }} \leq 2
\end{array}
$$

Thus, both the estimations for $D_{\text {tor }}^{\text {Lyap }}$ and $D_{\text {pol }}^{\text {Lyap }}$ uniquely indicate the irregularity of behavior of the model attractor. That can lead to the exponential growth of the initial error on the attractor.

\section{Statictics of the Lagrangian predictability}

The present section analyzes the predictability in general with uncertainty in the evolution law (the governing equations) and partially in initial conditions. Several physical factors can limit the model prediction in the Gulf of Mexico. First, the water inflow through the Yukatan Channel and the outflow through the Strait of Florida are described only approximately. The prediction error induced by inaccuracy in open boundary conditions can considerably reduce the model prediction time (Chu, 1999; Jiang and MalanotteRizzoli, 1999). Second, other sources of prediction errors are unresolved motions which are either parameterized in the model or neglected. Comparison between the drifter trajectories and modeled synthetic particle trajectories (Toner at al., 2001) demonstrates that after filtrating the effect of 30-hour tides, individual drifter trajectories are reproduced by the model with reasonable accuracy within 10 days. The present study utilizes the same drifter observations and numerical 


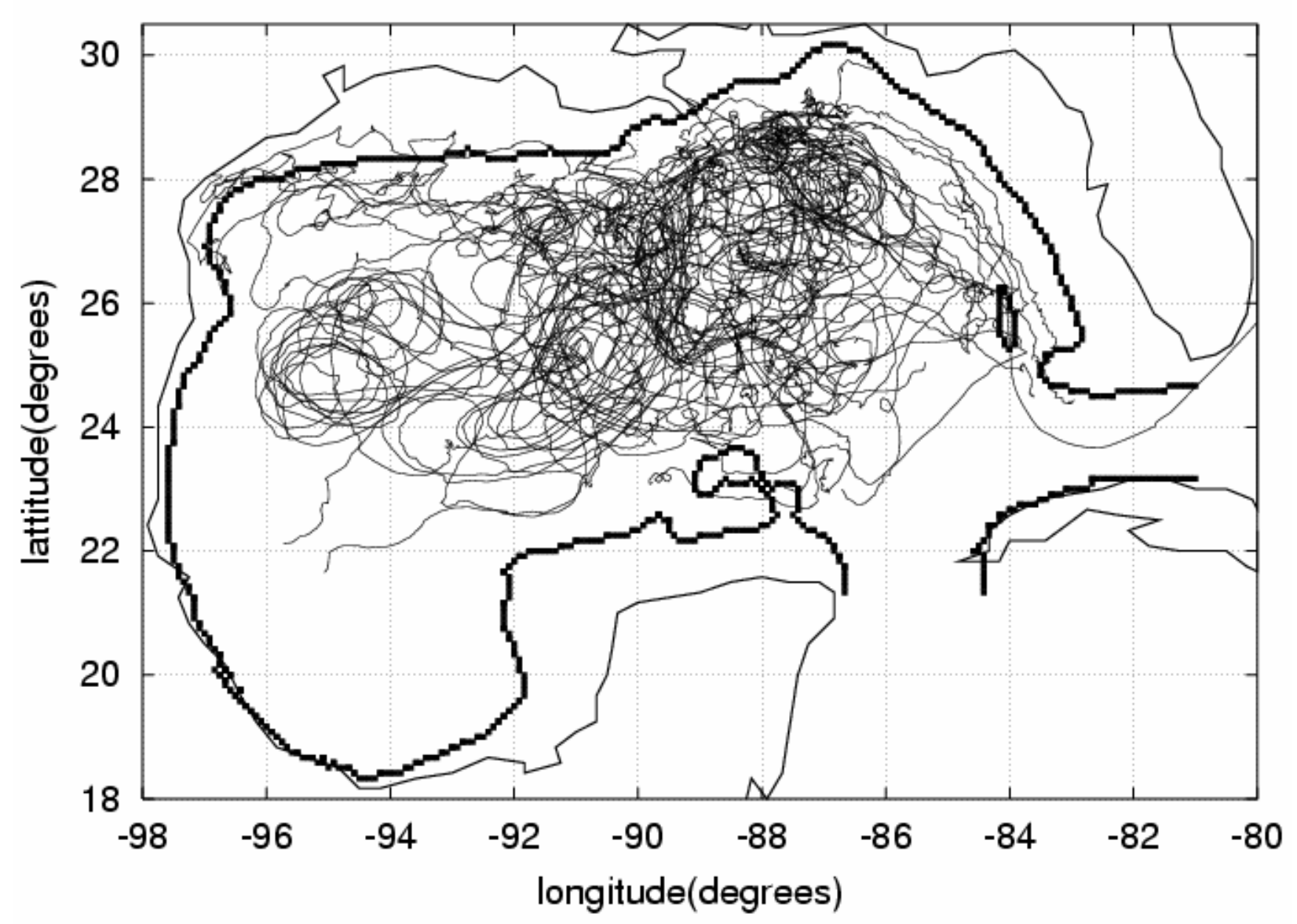

Fig. 10. Spaghetti of 55 drifting buoys used in the analysis.

model. However, in comparing with Toner et al. (2001) and Kuznetsov et al. (2002) the primary focus of our study is on statistical laws of the model prediction error growth.

\subsection{Drifter observations}

Fifty five satellite-tracked sonobuoys, manufactured by the Applied Technology Associates and deployed by the Horizon Marine, have provided the Lagrangian data used for the study. A nylon drogue at $50 \mathrm{~m}$ depth is tethered to the buoy hull that is $0.64 \mathrm{~m}$ in the water and $0.33 \mathrm{~m}$ in the air. Louisiana State University's Coastal Studies Institute/Earth Scan Laboratory tracked the buoys for the Horizon Marine. The drifter trajectories used in the present study are given in Fig. 10. The particle trajectories are obtained through timeintegration of the modeled horizontal velocity field at $50 \mathrm{~m}$ depth with the bi-cubic spline interpolation in space and the second-order interpolation in time.

\subsection{Model prediction error}

The differences of position $(\delta X, \delta Y)$ and velocity $(\delta U, \delta V)$ for any drifter-synthetic particle pair are stochastic. The ensemble average of the velocity difference over the trajectories $(\langle\delta U\rangle,\langle\delta V\rangle)$ shows weak model bias. For example, the bias $\langle\delta U\rangle$ has a minimum value of $-2.5 \mathrm{~cm} / \mathrm{s}$ on Julian Day-4 in 1998 and a maximum value of $5 \mathrm{~cm} / \mathrm{s}$ on Julian Day-18 in 1998 (Fig. 11a). However, the absolute RMS errors of velocity components (over the trajectories) are not small. For example, $\left\langle\delta U^{2}\right\rangle^{1 / 2}$ fluctuates between $34.5 \mathrm{~cm} / \mathrm{s}$ and $44.4 \mathrm{~cm} / \mathrm{s}$, and $\left\langle\delta V^{2}\right\rangle^{1 / 2}$ fluctuates between $31.7 \mathrm{~cm} / \mathrm{s}$ and $38.0 \mathrm{~cm} / \mathrm{s}$ (Fig. 11b). The variances of prediction error for both the drifter velocity components become practically constant after 4 days from the start of particle movement. In general large stationary variances of MPE indicate that the error evolutes nonlinearly (Nicolis, 1992; Chu et al., 2002a).

Let us discuss how the position model error characterizing differences between the drifter and particle positions evolves. We will determine its statistics by the first ten moments

$L_{q}(t)=\left\langle|\delta \tilde{\mathbf{Z}}|^{q}\right\rangle \quad q=1, \ldots, 10$,

where $\delta \tilde{\mathbf{Z}}=\{\delta X, \delta Y\}^{t}$.

It was pointed out by Chu et al. (2002a) that Eq. (30) holds a power law for $q=1$ and $q=2$. Here, we check the existence of a more universal power law for $q=1, \ldots, 10$

$L_{q}(t) \sim t^{\gamma_{q}}$

and specify the power exponent $\gamma_{q}$. 

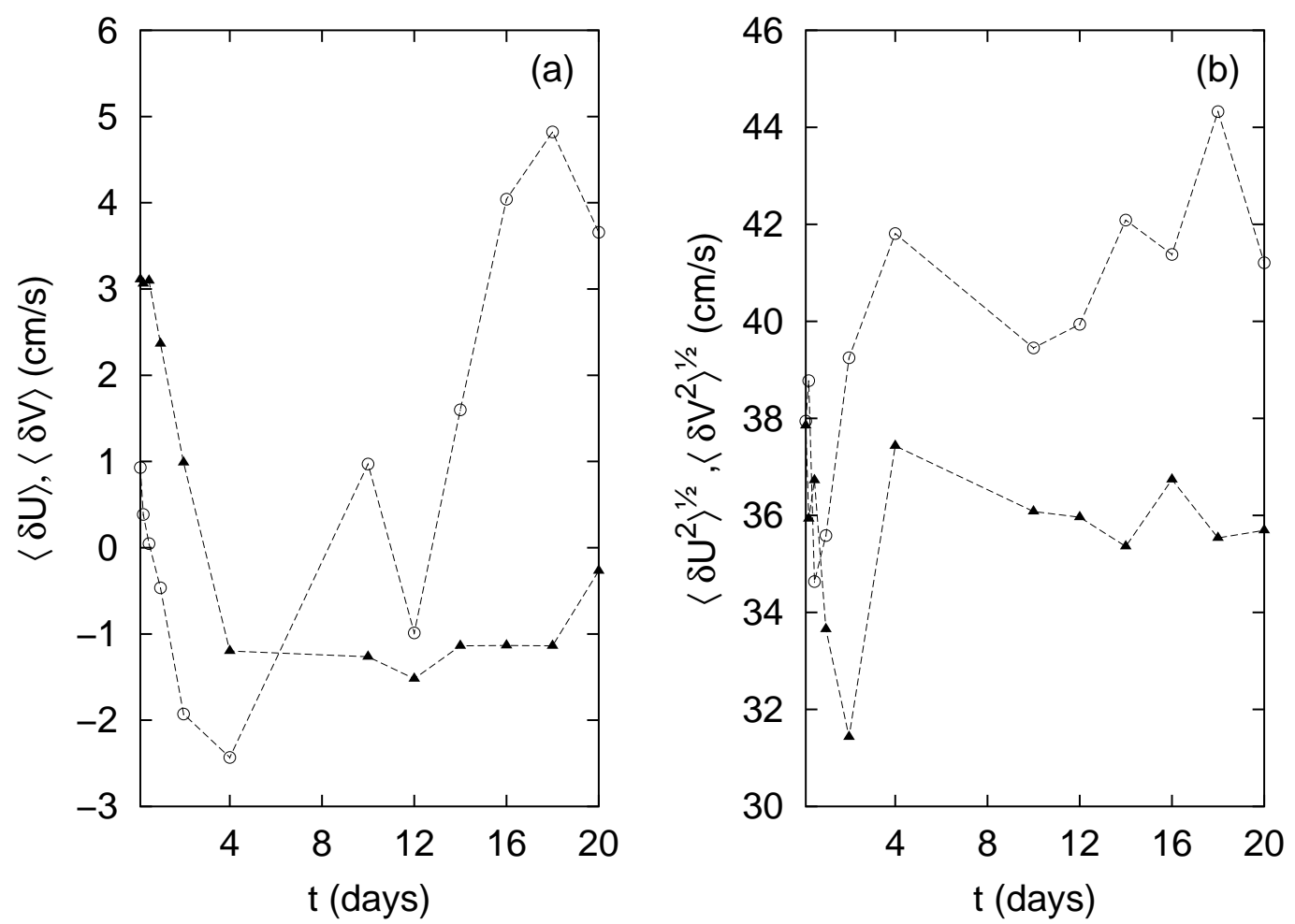

Fig. 11. Mean (a) and variance (b) of difference between buoy and particle velocities.

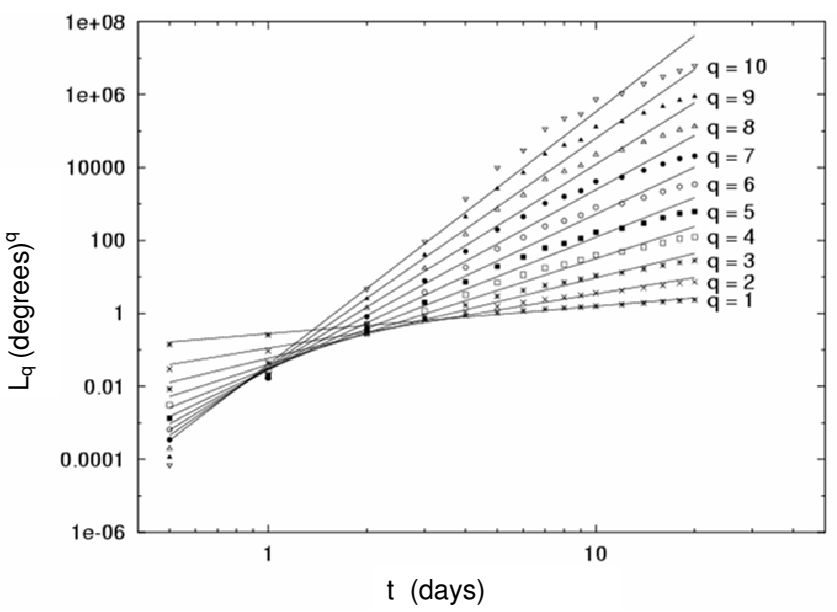

Fig. 12. The first ten moments of MPE. The solid lines represent the power laws which approximate computation results displayed by dots, crossings, triangles and squares.

If $\gamma_{q}=\mu q / 2$,

the PDF of MPE has a self-similar form. MPE described by relation (31) with (32) is a Gaussian (non-Gaussian) process if $\mu=1(\mu \neq 1)$ (del Castillo-Negrete, 1998). Any deviation from relation (32) suggests that the MPE is an intermittent process.

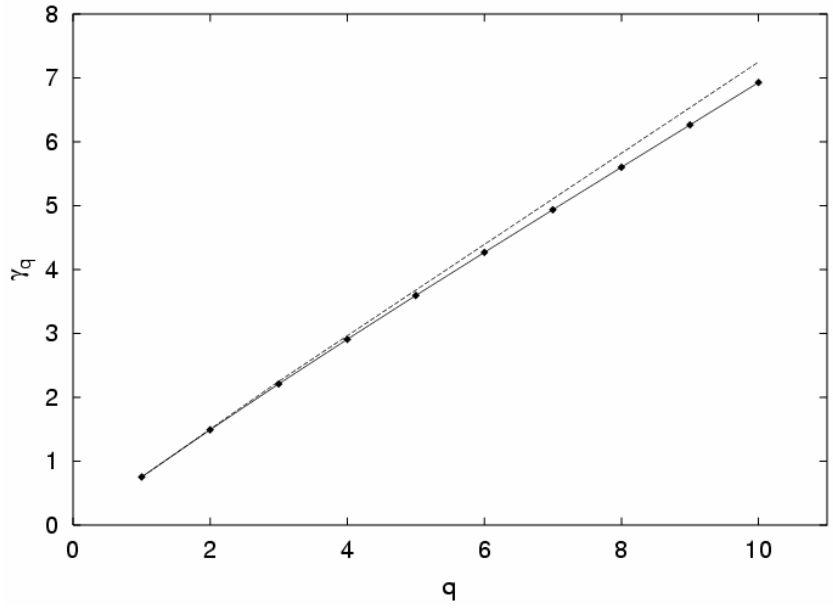

Fig. 13. The scaling exponent $\gamma_{q}$ as measured within the range $12 \mathrm{~h}<t<20$ days. Dashed line is the $3 q / 4$ law. Solid line is the best fit. Difference between the $3 q / 4$ law and the best fit observed for the moments numbered higher than 4 is explained by reducing accuracy of computational results from finite sampling time effects.

Our computations display a power dependence of $L_{q}(t)$ on the time $t$ ( $12 \mathrm{~h}<t<20$ days) from $q=1$ to $q=10$ (Fig. 12):

$L_{q}(t) \sim t^{\mu q / 2}$

with

$\mu \approx 1.49 \pm 0.03$, 

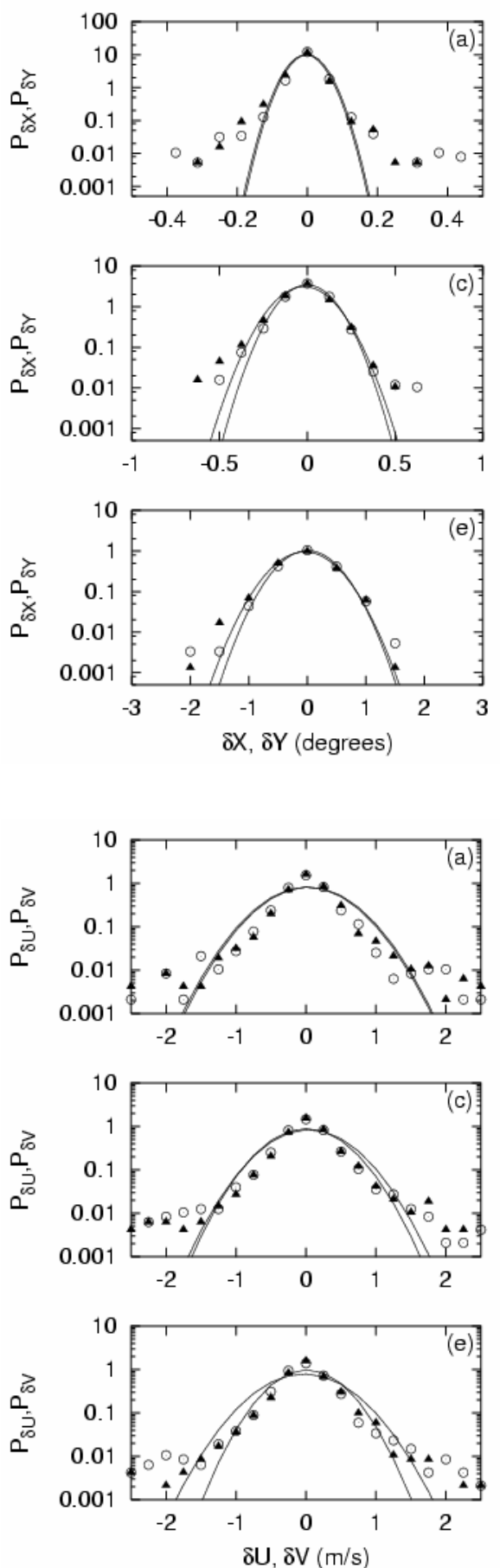
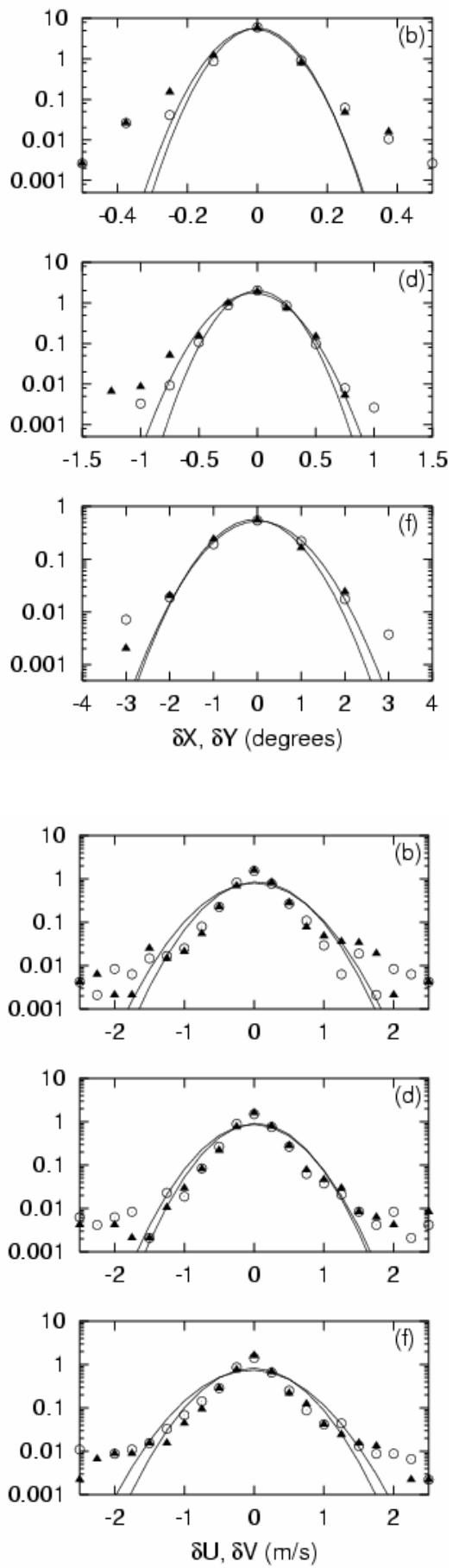

Fig. 14. $P_{\delta X}=\bar{P}_{1}(\delta X, 0, t)$ (the black triangles) and $P_{\delta Y}=\bar{P}_{1}(0, \delta Y, t)$ (the white dots) through $3 \mathrm{~h}(\mathbf{a}), 6 \mathrm{~h}(\mathbf{b})$,

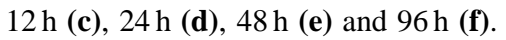
The solid lines are Gaussian approximations.
Fig. 15. $P_{\delta U}=\bar{P}_{2}(\delta U, 0, t)$ (the black triangles) and $P_{\delta V}=\bar{P}_{2}(0, \delta V, t)$ (the white dots) through $3 \mathrm{~h}$ (a), $6 \mathrm{~h}$ (b),

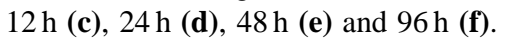
The solid lines are Gaussian approximations.
To assess the possible impact of finite sample size effects on estimating $\mu$, we apply a simple test that was proposed earlier by Tennekes and Wijngaard (1972). The maximum error for estimating $\mu$ is about $0.02-0.03$ for the high-order moments. For the first four moments $L_{q}(q=1, \ldots, 4)$ the scaling exponent is very close to $3 q / 4$ (Fig. 13).

The power growth of MPE is interpreted in terms of the normal and anomalous diffusion (del Castillo-Negrete, 1998). The cases of $\mu=1$ and $\mu>1$ may be called as "the normal diffusion" and "the super-diffusion" regimes of MPE growth, respectively.

\subsection{PDF of MPE}

To understand how the statistics of $\delta \tilde{\mathbf{Z}}$ departures from Gaussian distribution, the following shorted PDFs are applied: $\bar{P}_{1}(\delta X, \delta Y, t)$ and $\bar{P}_{2}(\delta U, \delta V, t)$. The appropriate crosssections of these PDFs are plotted in Figs. 14 and 15 displaying their explicit symmetry and isotropy. $\bar{P}_{1}(\delta X, \delta Y, t)$ has a non-Gaussian shape for various times from $3 \mathrm{~h}$ to 10 days. The departures from Gaussian behavior and the presence of tails stretched in large MPE, are both evident in these figures. At times longer than 10 days this function tends to a 

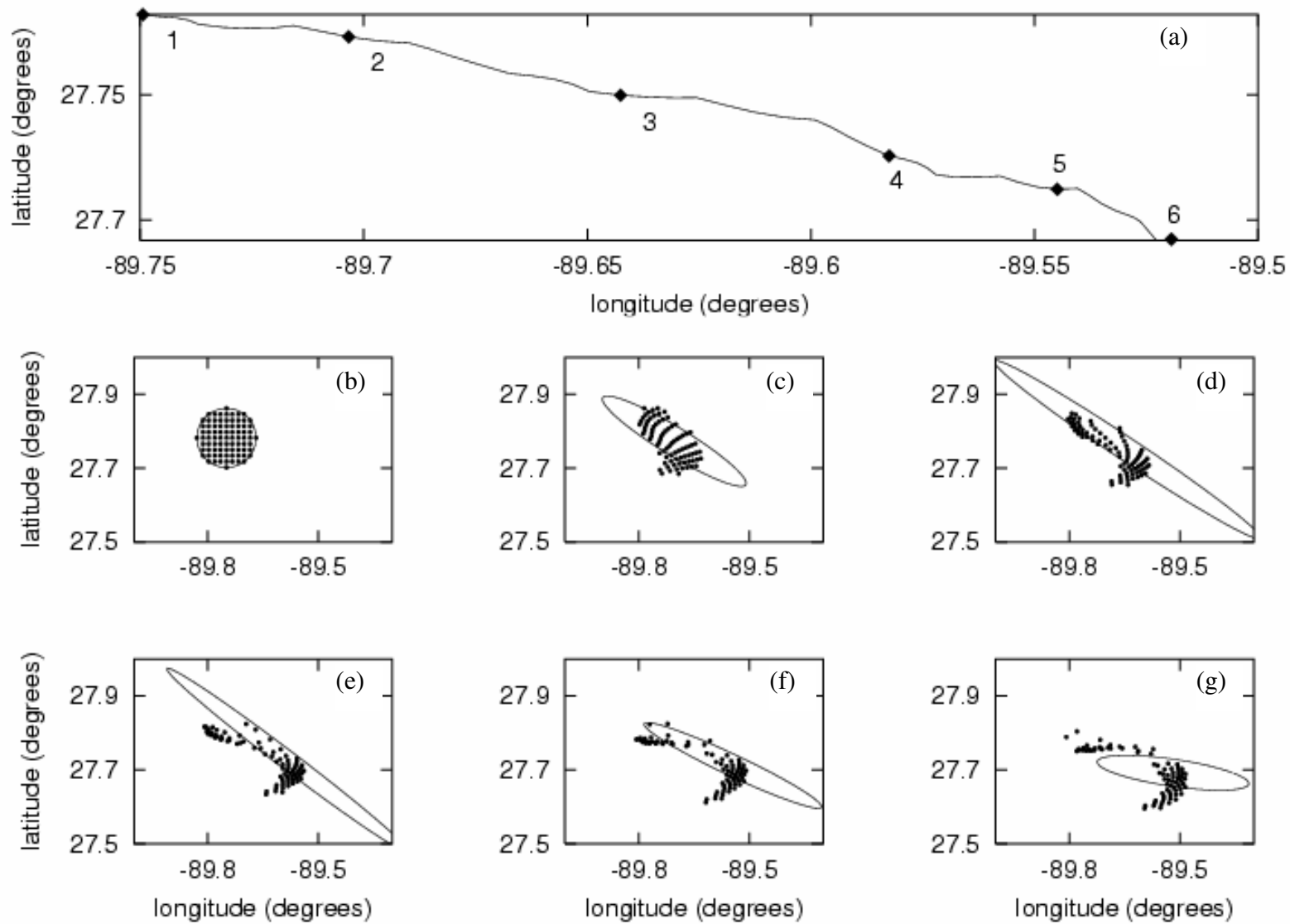

Fig. 16. Cluster spread along the trajectory (a) of H17543 drifter. Positions 1, 2, 3, 4, 5, 6 of cluster center (a) and corresponding to them positions of the cluster (b), (c), (d), (e), (f), (g) are given through every $8 \mathrm{~h}$. Initially the cluster is round with the radius equaled to $1 / 12^{\circ}$ (b). The solid lines are the cluster boundaries predicted by the singular vector approach. The black dots are particles.

Gaussian shape because of correlations for a pair of buoyparticle decay. The shape of $\bar{P}_{2}(\delta U, \delta V, t)$ practically does not change with time (Fig. 15).

Using the analogy between the pair-particle dispersion and the drifter-synthetic particle divergence, it is reasonable to assume that $\bar{P}_{1}(\delta X, \delta Y, t)$ holds the following diffusion-like equation (Boffeta and Celani, 2000):

$\frac{\partial P_{\delta R}}{\partial t}=\frac{\partial}{\partial R}\left(K_{\mathrm{eff}}(R, t) \frac{\partial P_{\delta R}}{\partial R}\right)$,

where $\delta R=\sqrt{\delta X^{2}+\delta Y^{2}}, \quad K_{\text {eff }}(R, t) \sim R^{3 / 4}$ is the Richardson-Obukhov type effective diffusion coefficient.

The solution of Eq. (34) is written as (Klafer et al., 1987)

$P_{\delta R} \cong t^{-9 / 2} \exp \left(-C \cdot R^{2 / 3} / t\right)$,

here, $C$ is constant.

Relation (35) correctly describes the evolution of the shorted PDF of MPE for the small and intermediate times. This indicates that the statistics of the Lagrangain prediction error in the case of the second kind of predictability (Lorenz, 1984) can be described by diffusion-like equations. Note that the model error is scaled within the time intervals from $12 \mathrm{~h}$ to 20 days. These time scales correspond to the spatial scales varying from $20 \mathrm{~km}$ to $300 \mathrm{~km}$.

\subsection{Linear tangent model}

To examine applicability of linear tangent models for the analysis of Lagrangian predictability of high-resolution regional models we study the dispersion of small-size particle clusters with the initial round shape and the radius equaled to $1 / 12^{\circ}$ along the drifter trajectories $\left[\boldsymbol{Z}_{\mathrm{ref}}(t)=\boldsymbol{Z}(t)\right]$ and compare computation results with predictions from the singular vector approach.

If a linear tangent model is valid within the reference pe$\operatorname{riod}\left(T-t_{0}\right)$, the cluster rapidly gets an ellipsoidal shape conserved within this period. In opposite case, the cluster is quickly deformed to a pancake-like shape. The evolution of the cluster shape can be studied in two ways: through direct modeling and by the singular vector analysis. This allows estimating the reference period for the real circulation model from the real oceanographic observations.

The results discussed above say that the Lagrangian model skill is non-Gaussian even for small times. Thus, we can not expect large reference periods. However, their exact estimations are of considerable interest for the practical predictability.

In general we found that linear tangent models can be applied to approximate the MPE growth within very short time 

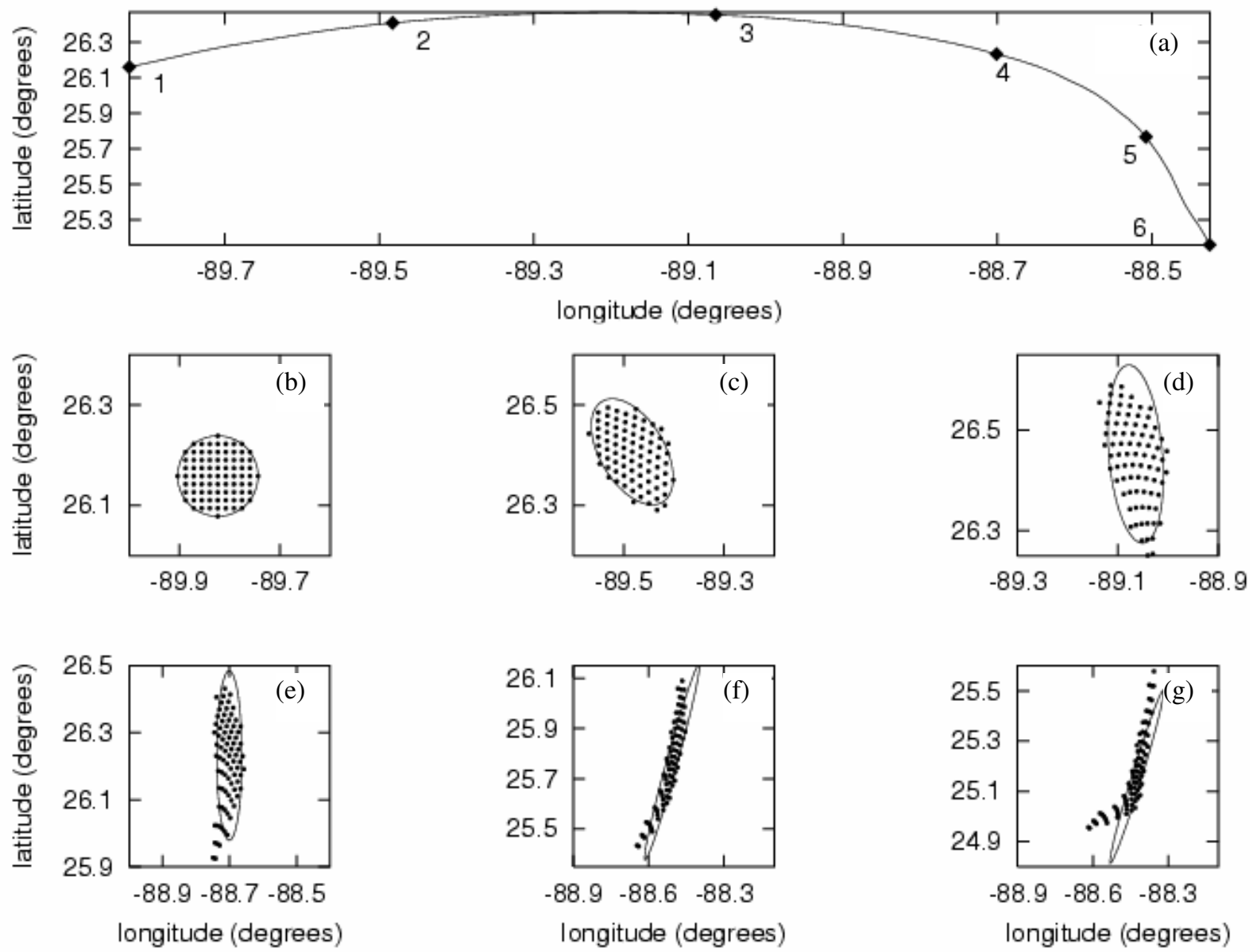

Fig. 17. Cluster spread along the trajectory (a) of H17547 drifter. Positions 1, 2, 3, 4, 5, 6 of cluster center (a) and corresponding to them positions of the cluster (b), (c), (d), (e), (f), (g) are given through every $15 \mathrm{~h}$. Initially the cluster is round with the radius equaled to $1 / 12^{\circ}$ (b). The solid lines are the cluster boundaries predicted by the singular vector approach. The black dots are particles.

intervals limited by several days. As an example, two drifter (H17543 and H17547) trajectories are selected to show this (Figs. 16 and 17). The reference periods for H17543 and H17547 drifters were estimated as 8 and $30 \mathrm{~h}$, respectively. The mean reference period $\left\langle\left(T-t_{0}\right)\right\rangle$ was equaled to $21 \mathrm{~h}$, i.e. after this time a linear tangent model breaks down. Thus, $21 \mathrm{~h}$ is the practical limit to apply a linear tangent model of MPE in the Gulf of Mexico.

The analysis of evolution of all clusters used to estimate the reference period also demonstrate that although a cluster is quickly deformed to a pancake-like shape, the cluster center moves along the drifter trajectory during considerably larger time than the reference period, i.e. the local model drift was a quite small.

Because the mean reference period should be limited only by $21 \mathrm{~h}$, we will not apply linear tangent models for the analysis of Lagrangian predictability any more.

\subsection{IT statistics}

The PSP (see Eq. 7) with zero initial errors, $F(t, \epsilon)$, is calculated from the drifter and particle trajectories with the tolerance level ranging from $0.05^{\circ}$ to $1.25^{\circ} . F(t, \epsilon)$ for four dif- ferent tolerance levels $\left(0.25^{\circ}, 0.5^{\circ}, 0.75^{\circ}\right.$, and $\left.1.25^{\circ}\right)$ clearly shows non-Gaussian distribution (Fig. 18). The long tails stretched into large ITs demonstrate the existence of the longterm correlations between the drifters and synthetic particles. We see that individual Lagrangian trajectories can be predicted within 20-25 day period even if the mean Lagrangian prediction time does not exceed $4-5$ days. Individual predictions with abnormal large prediction periods were called the extremely successful predictions (ESP) (Chu et al., 2002a).

The PSP tails (Fig. 18) have the power behavior for the long IT period with the power exponents as $(2.17 \pm 0.07,1.98 \pm 0.09,2.08 \pm 0.10,1.77 \pm 0.25)$ for $\epsilon$ as $\left(0.25^{\circ} ; 0.5^{\circ}, 0.75^{\circ}, 1.25^{\circ}\right)$, respectively. That identifies the power exponent as larger than 2 .

If the power exponent is larger than 2, the mean and variance of IT exist (Fig. 19). Both these values are scaled as

$$
\left\langle\tau_{\text {irrev }}\right\rangle \sim \epsilon^{\alpha}, \quad\left\langle\tau_{\text {irrev }}^{2}\right\rangle \sim \epsilon^{\beta}
$$

with $\alpha \approx 1.21 \pm 0.11$ and $\beta \approx 2.17 \pm 0.15$.

That indicates non-Gaussian feature of the Lagrange prediction skill. It was pointed out by Popov (1970) that $\alpha=1$ and $\beta=2$ for a Gaussian statistics. 

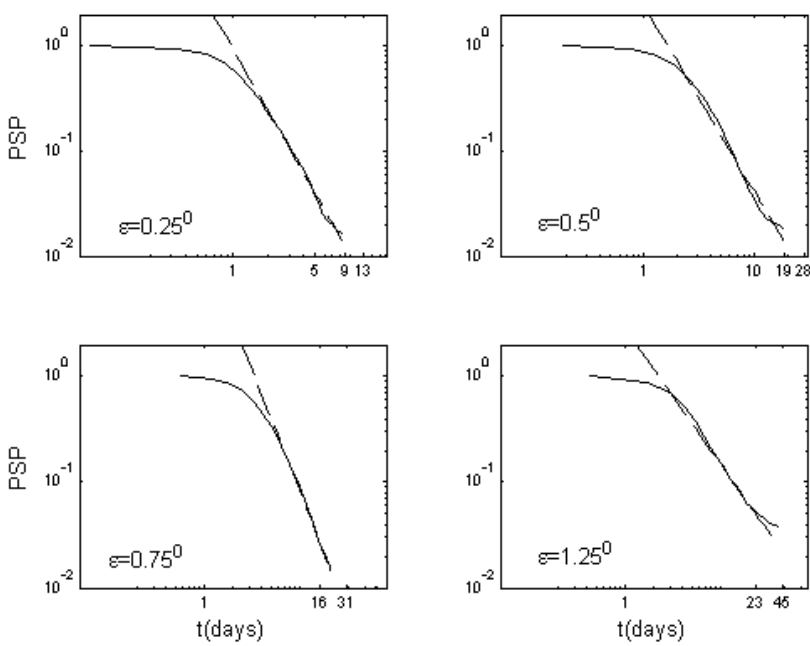

Fig. 18. PSP of IT calculated for different levels of tolerance $\epsilon$. Solid and dashed lines are the PSP and the proposed theoretical power laws to approximate it for large times.

The mean irreversible time can also be easily computed from model (34), (35) and through Popov's (1970) method. The theoretical estimation of $\alpha$ is $4 / 3$. We see that the best fit of the power exponent estimated as $1.21 \pm 0.11$ agrees with the theoretical value very well.

\section{Reconstruction of model attractor}

It was demonstrated above that mean Lagrangian prediction skill of high-resolution oceanographic models is not very high, up to 4-5 days, and models predict the real drifter trajectories only within short and intermediate time periods. A key question is: may the spatial structure of such a model attractor be reconstructed from the rare drifter observations?

An approach developed in Eremeev et al. (1992) and Chu et al. (2003a,b) allows reconstructing velocity field from sparse and noisy observations. Accordingly to them a velocity field is projected onto $N$ dimension phase space generated by some basis functions. Then, these projections (the reconstruction coefficients) are estimated from $P$ observations. In general the reconstruction procedure requests that $P>(1.5-2) N$.

We will apply this approach for estimating the Gulf of Mexico model attractor structure from 15 drifter buoys if EOFs computed from model circulation are taken as the basis functions.

Although the EOF decomposition has rapid convergence by the energetic norm, the accurate approximation of spatial structure of circulation needs more than few EOFs. The accuracy of the EOF truncation is represented by the relative RMS errors of the toroidal and poloidal potentials

$$
\begin{aligned}
& \chi_{\text {tor }}(t, N)=\left\|\Psi^{\prime}\right\|^{-1}\left\|\Psi^{\prime}-\Psi_{N}^{\prime}\right\| \\
& \chi_{\mathrm{pol}}(t, M)=\left\|\Phi^{\prime}\right\|^{-1}\left\|\Phi^{\prime}-\Phi_{M}^{\prime}\right\|
\end{aligned}
$$

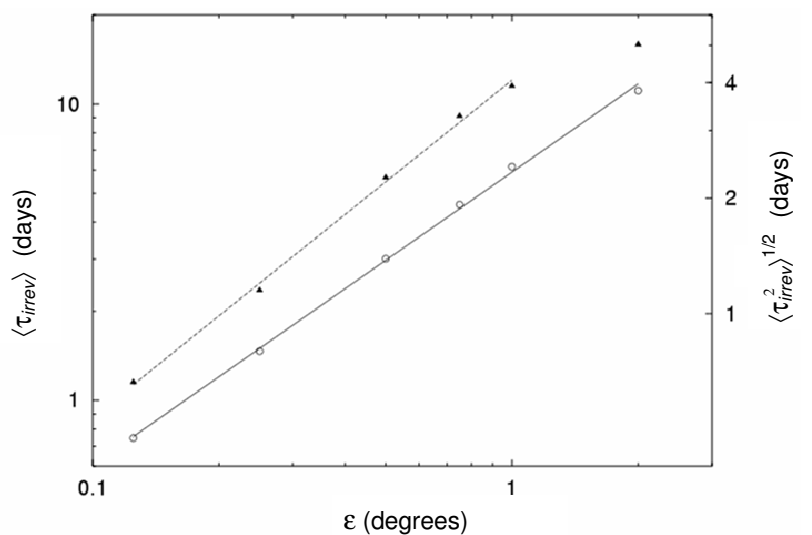

Fig. 19. The mean IT (white dots) and its variance (black triangles) as functions of the tolerance $\epsilon$.

where the Euclidian norm \|\| summarizes over all points of the model computation grid, $\Psi_{N}^{\prime}=\sum_{n=1}^{N} A_{n} \Psi_{n}$ and $\Phi_{M}^{\prime}=\sum_{m=1}^{M} B_{m} \Phi_{n}$ are the pulsations of potentials approximated by $N$ toroidal and $M$ poloidal modes, respectively.

Figures 20 and 21 show the temporally varying $\chi_{\text {tor }}(t, N)$ and $\chi_{\text {pol }}(t, M)$ with six different mode truncations $(1,3,6$, $10,30,50)$, respectively. For accurate representation of $\Psi^{\prime}$ and $\Phi^{\prime}$, large number of mode truncation is needed. For example, for the RMS error of the toroidal potential $\left(\chi_{\text {tor }}\right)$ to be less than $1 \%, 50$ toroidal modes are needed.

Because we utilized only 15 buoys traced the Gulf of Mexico circulation, not more than 10-12 reconstruction coefficients can be estimated from these observations. To satisfy this requirement the reconstruction was provided on $186 \mathrm{Ju}-$ lian Day of model calculations because at this time we can neglect the poloidal current and approximate the toroidal potential through 10 toroidal modes with the accuracy of $2.5 \%$. The second reason of such a choice is that the reconstruction error caused by heterogeneity of drifter covering of the area of interest was considerably reduced. For further discussions see Chu et al. (2003a, b).

To reconstruct a circulation pattern we need to estimate mode amplitudes $A_{1}, \ldots, A_{10}$ from Lagrangian data, i.e. from knowledge of drifter velocity $(U, V)$ such that

$$
\begin{aligned}
\| U & -\nabla_{y}\left(\bar{\Psi}+\Psi^{\prime}\right) \|_{P}^{2} \\
& +\left\|V+\nabla_{x}\left(\bar{\Psi}+\Psi^{\prime}\right)\right\|_{P}^{2} \rightarrow \min
\end{aligned}
$$

where the summation in the Euclidian norm is over all drifter positions. The reconstruction coefficients $A_{1}, \ldots, A_{10}$ were computed by the Singular Value Decomposition method (Engl, et al., 1996).

Figure 22 shows the model circulation at 186 Julian Day, velocities calculated from the drifter observations for the same time, the reconstructed currents and residual circulation, i.e. difference between the model and reconstructed currents. It is noted from comparison of Figs. 22a and 22c 

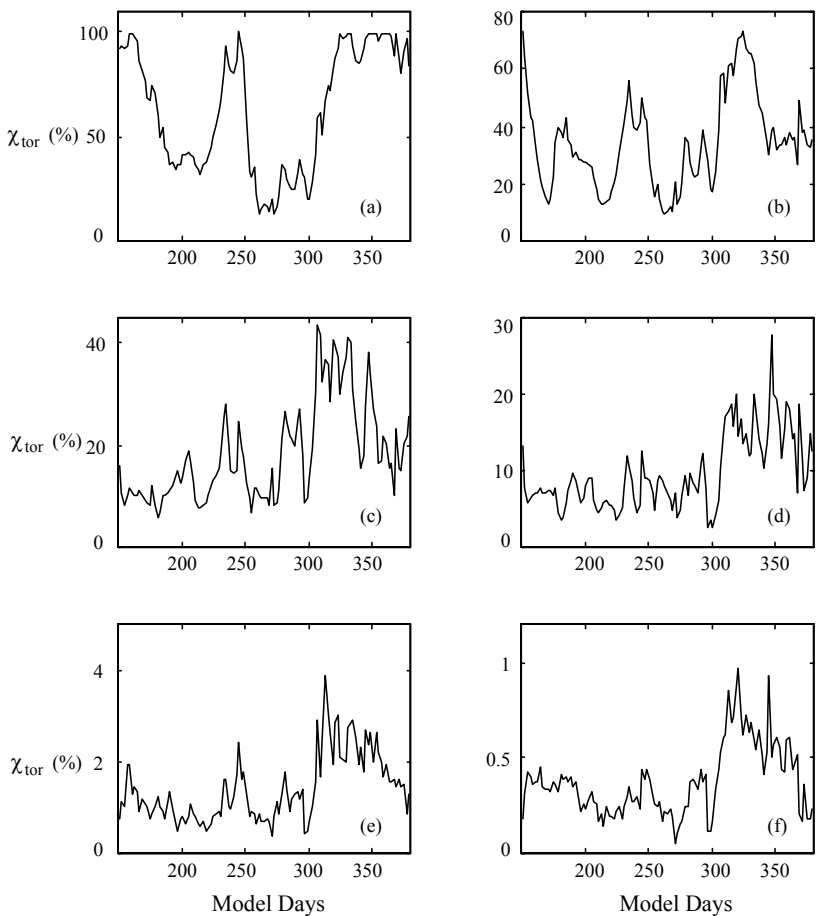

Fig. 20. RMS errors $\left(\chi_{\text {tor }}\right)$ of approximation of the toroidal circulation through 1 (a), 3 (b), 6 (c), 10 (d), 30 (e) and 50 (f) toroidal EOFs.

that the spatial structure of circulation is consistent in different scales (the Loop Current and eddies) between the reconstructed and model fields. The residual velocity, i.e. the reconstruction error, is quite high in the central part of the Gulf of Mexico (Fig. 22d). The mean relative RMS error between the model and reconstructed fields is about $28 \%$.

\section{Conclusions}

(1) Lagrangian predictability of the high-resolution Gulf of Mexico nowcast/forecast system developed at the University of Colorado at Boulder, was examined using 55 Lagrangian drifters. The MPE of drifter positions and velocities was estimated through RMS errors and irreversible-skill time. The approach based on the linear tangent model was found unsatisfactory because in mean the reference period can not be taken longer than 1 day. Obviously that such short reference periods are of no interest for the practical applications.

(2) The model attractor at $50 \mathrm{~m}$ depth was analyzed through EOF decompositions developed for toroidal (nondivergent) and poloidal (divergent) components of circulation. Lyapunov dimensions estimated by the phenomenological method of Syrovich (1989) held the following inequalities

$15 \leq D_{\text {tor }}^{\text {Lyap }} \leq 16 \quad 1 \leq D_{\text {pol }}^{\text {Lyap }} \leq 2$

That evidences an irregular behavior of energetic and spectral characteristics of the circulation and probably, exponen-
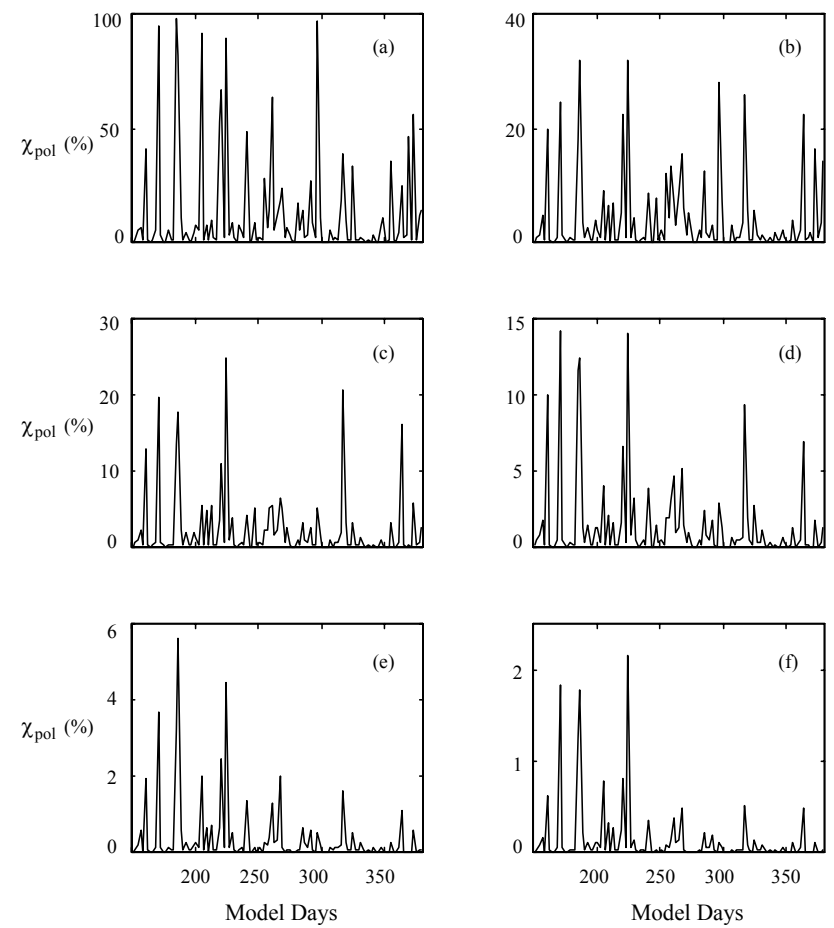

Fig. 21. RMS errors $\left(\chi_{\text {pol }}\right)$ for the poloidal circulation approximated through 1 (a), 3 (b), 6 (c), 10 (d), 30 (e) and 50 (f) poloidal EOFs.

tial growth of prediction error caused by uncertainty in initial conditions utilized by the model.

(3) We pointed out that three scenarios are possible in a model-drifter comparison.

(i) The long-term (global) predictability is referred when the model reproduces the circulation attractor (the robust regime) in small-scale details and predicts the real drifter trajectory within large time intervals.

(ii) In the short and intermediate predictability regime the model correctly simulates the circulation attractor but not all small-scale circulation details. The drifter trajectories can also be predicted only locally.

(iii) The model approximates the drifter trajectories only within short time periods (the partial predictability). Our estimations selected the second scenario as one realized for the Gulf of Mexico model.

(4) Statistics of MPE is nonGaussian for short, intermediate and long times.The MPE grows by the power law with exponent equaled to $3 / 2$ in the range $12 \mathrm{~h}<t<20$ days, that corresponds to spatial scales between $20 \mathrm{~km}$ and $300 \mathrm{~km}$. Accordingly to terminology of the theory of anomalous diffusion (Klafer et al., 1987) we called this dynamical regime as "the super-diffusion" growth of prediction error. Obviously that such a growth indicates the fast displacement in a pair of buoy-particle in areas where the fixed points like as saddles may be identified in the topology structure of modeling circulation. In our opinion the power law of model prediction 

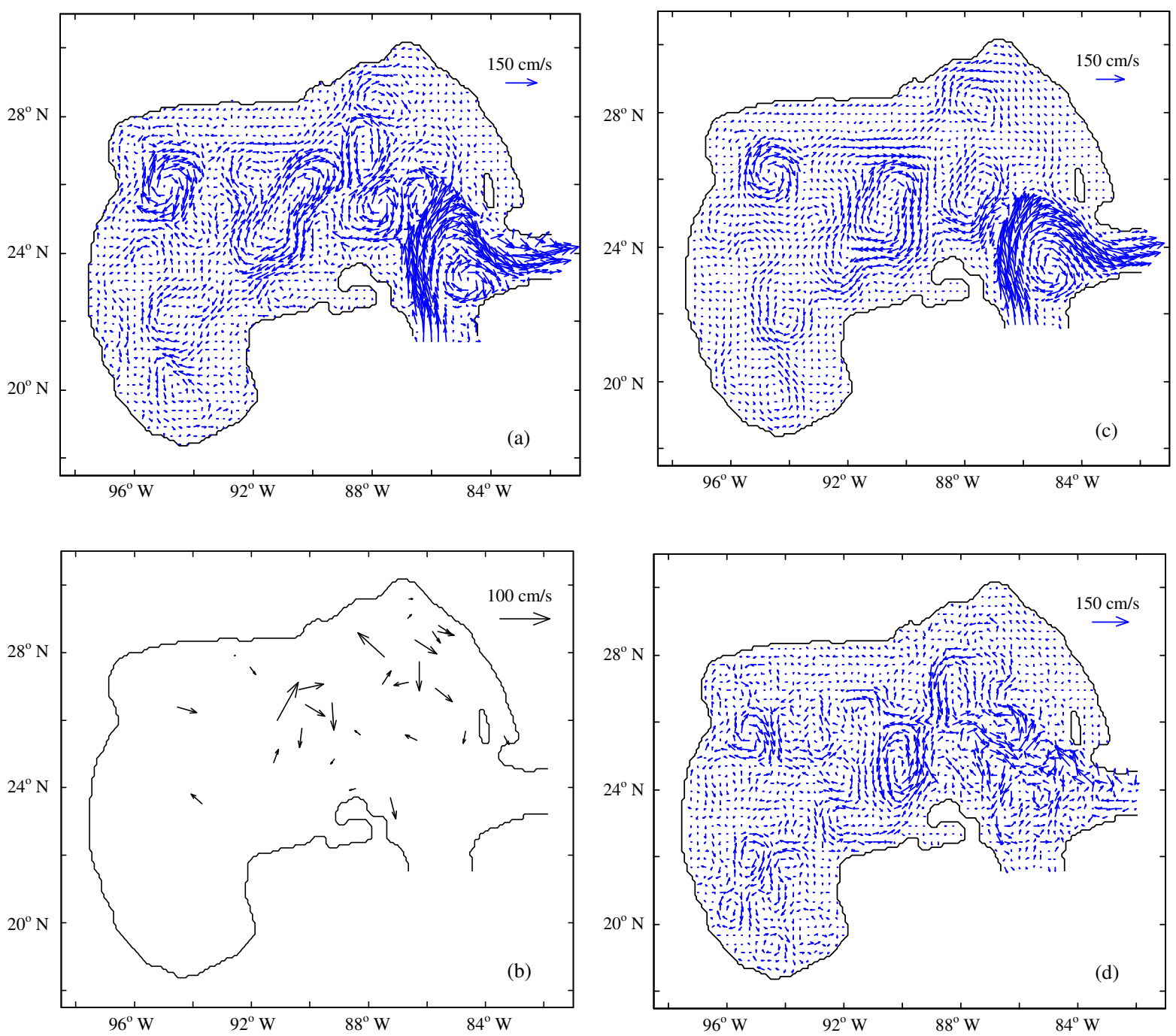

Fig. 22. Model circulation on 186 Julian Day (a), velocities calculated from the drifters for the same time (b), the reconstructed circulation (c) and the residual circulation (d).

error obtained for a high-resolution model in the specific geographic region may be found for other models and marine areas. As examples, the model prediction error grows along a power law for POP model of the North Atlantic (Ivanov et al., 2003).

The $q-$ th moment of MPE is scaled with power exponent of $3 q / 4$. That in general says about the self-similar feature of model prediction skill. The PDF of MPE holds the diffusionlike Eq. (34). Universality of the parameterization of Eq. (34) to describe the growth of prediction error for other high resolution numerical models and for other geographic regions will be examined in a separate paper.

(5) The PSP clearly shows a long tail stretched into large ITs. This tail is a signature of long-term correlations between the drifter and particle trajectories in separate individual pairs of drifter-particle. Individual Lagrangian trajectories can be predicted within a 20-25 day period even if the mean Lagrangian prediction time does not exceed 4-5 days. The best fit of the power exponent estimated as $1.21 \pm 0.11$ agrees very well with the theoretical value of 1.33 obtained from the diffusion-like MPE model.

(6) The obtained results has the following practical capability. First, statistics of the mean prediciton errors can be utilized to develop numerical scheme for Lagrangian data assimilation. That will be done in a separate paper.

Second, in general we can distinguish between different approaches in study of ocean: Eulerian (in terms of velocity fields) and Lagrangian (in terms of trajectories of fluid particles). Even through these two points of view are in principle equivalent, the relationship between predictabilities as seen in the realm of two approches, is still an open problem and there is no evidence of a fixed correspondence between Eulerian and Lagrangian chaotic behaviours.

It was pointed out in Eremeev et al. (1992) that if the Lagrangian correlation scale $T_{L}$ determined from the Lagrangian data exists and drifting buoy movements are 
described as pure stochastic, a model in mean may not predict Lagrangian trajectories for time intervals $\left(\tau_{\text {pred }}\right)$ longer than this scale, i.e. $\tau_{\text {pred }}<T_{L}$. In contrast, if drifter movements are chaotic (Lagrangian chaos) we may select models allowing to predict longer that $T_{L}$ (Kravtsov, 1989). A practical procedure of similar selection was developed by Pires et al. (1996).

\section{Appendix A Computation of the singular values}

Let us introduce the matrix $\boldsymbol{Q}=\boldsymbol{L}^{-1}$ and differentiate Eq. (13) with respect to $t$ :

$$
\begin{aligned}
&-\left(\boldsymbol{Q}^{-1}\right.\left.\frac{d \boldsymbol{Q}}{d t} \boldsymbol{Q}^{-1} \delta \boldsymbol{Z}, \delta \boldsymbol{Z}\right) \\
&+\left(\boldsymbol{Q}^{-1} \frac{d \delta \boldsymbol{Z}}{d t}, \delta \boldsymbol{Z}\right)+\left(\boldsymbol{Q}^{-1} \delta \boldsymbol{Z}, \frac{d \delta \boldsymbol{Z}}{d t}\right)=0
\end{aligned}
$$

Using Eq. (11) Eq. (A.1) is rewritten as

$$
\begin{aligned}
& \left(\boldsymbol{Q}^{-1}\left[\frac{d \boldsymbol{Q}}{d t}-\boldsymbol{J}_{1} \boldsymbol{Q}-\boldsymbol{Q} \boldsymbol{J}_{1}^{t}\right] \boldsymbol{Q}^{-1} \delta \boldsymbol{Z}, \delta \boldsymbol{Z}\right)=0 \\
& \text { or } \quad \frac{d \boldsymbol{Q}}{d t}=\boldsymbol{J}_{1} \boldsymbol{Q}+\boldsymbol{Q} \boldsymbol{J}_{1}^{t}
\end{aligned}
$$

with obvious initial conditions

$$
\boldsymbol{Q}\left(t_{0}\right)=\boldsymbol{Q}_{0}
$$

where, $\boldsymbol{Q}_{0}$ is a non-zero matrix measuring uncertainty of model initial conditions.

So, for the given reference period $T-t_{0}$, the diagonal elements of the matrix $\boldsymbol{Q}$ determine the size of ellipsoid of prediction uncertainty. Non-diagonal matrix elements identify a position of this ellipsoid in space. Obviously, the geometrical center of the ellipsoid moves along the reference trajectory $\boldsymbol{Z}_{\text {ref. }}$. The eigenvalues and eigenfunctions of the matrix $\boldsymbol{Q}$ are easily recalculated in the traditional singular values and vectors by way of simple algebraic manipulations.

Acknowledgements. This work was supported by the Office of Naval Research, Naval Oceanographic Office, and the Naval Postgraduate School. L. H. Kantha acknowledges with pleasure the partial support by the ONR under grants ONR N 00014-02-1-1043 and $\mathrm{N}$ 00014-03-1-0488. This work was supported by the NATO linkage grant for L. M. Ivanov, T. M. Margolina and O. V. Melnichenko. Detailed and useful comments of an anonymous referee are gratefully acknowledged.

Edited by: S. Wiggins

Reviewed by: two referees

\section{References}

Acero-Schertzer, C. E., Hansen, D. V., and Swenson, M. S.: Evaluation and diagnosis of surface currents in the National Centers for Environmental Prediction's ocean analyses, J. Geophys. Res., 102, 21037-21048, 1997.
Aubry, N., Guyonnet, R., and Lima, R.: Spatiotemporal analysis of complex signals: theory and applications, J. Stat. Phys., 64, 3/4, 683-739, 1991.

Boffeta, G. and Celani, A.: Pair dispersion in turbulence, Physica A, 280, 1-9, 2000.

Chu, P. C.: Two kinds of predictability in the Lorenz system, J. Atmos. Sci., 56, 1427-1432, 1999.

Chu, P. C., Ivanov, L. M., Kantha, L. H., Melnichenko, O. V., and Poberezhny, Yu. A.: Power law decay in model prediction skill, Geophys. Res. Lett., 29, 15, 34-1-34-4, 2002a.

Chu, P. C., Ivanov, L. M., and Fan, C. W.: Backward Fokker-Planck equation for determining model valid prediction period, J. Geophys. Res., 107, C6, 10.1029/2001JC000879, 2002b.

Chu, P. C., Ivanov, L. M., Margolina, T. M., and Melnichenko O. V.: On probabilistic stability of an atmospheric model to various amplitude perturbations, J. Atm. Sci., 59, 2860-2873, 2002c.

Chu, P. C., Ivanov, L. M., Margolina, T. M., Korzhova, T. P., and Melnichenko, O. V.: Analysis of sparse and noisy ocean current data using flow decomposition, Part 1, Theory, J. Atmos. Ocean. Tech., 20, 478-491, 2003a.

Chu, P. C., Ivanov, L. M., Margolina, T. M., Korzhova, T. P., and Melnichenko, O. V.: Analysis of sparse and noisy ocean current data using flow decomposition, Part 2, Applications to Eulerian and Lagrangian data, J. Atmos. Ocean. Tech., 20, 492-512, $2003 b$.

Chu, P. C. and Ivanov, L. M.: Multiply prediciton regimes in regional ocean models, J. Phys. Ocean., (submitted), 2003c.

Davis, R. E., Killworth, P. D., and Blundell, J. R.: Comparison of Autonomous Lagrangian Circulation Explorer and fine resolution Antarctic model results in the South Atlantic, J. Geophys. Res., 101, C1, 855-884, 1996.

del Castillo-Negrete, D.: Asymmetric transport and non-Gaussian statistics of passive scalars in vortices in shear, Phys. Fluids, 10, 576-594, 1998.

Engl, H. W., Hanke, M., and Neubauer, A.: Regularization of Inverse Problems, Kluwer Academic Publishers, Dordrecht / Boston / London, 1996.

Eremeev, V. N., Ivanov, L. M., and Kirwan, A. D., Jr.: Reconstruction of oceanic flow characteristics from quasi-Lagrangian data 1.Approach and mathematical methods, J. Geophys. Res., 97, 9733-9742, 1992.

Falkovich, G., Gawedzki, K., and Vergassola, M.: Particles and fields in fluid turbulence, Rev. Mod. Phys., 73, 914-972, 2001.

Figueroa, H. A. and Olson, D. B.: Eddy resolution versus eddy diffusion in a double gyre GCM, Part I: The Lagrangian and Eulerian description, J. Phys. Ocean., 24, 371-402, 1994.

Gardiner, C. W.: Handbook of Stochastic Methods for Physics, Chemistry and the Natural Sciences, Springer-Verlag, 1985.

Garraffo, Z. D., Mariano, A. J., Griffa, A., Veneziani, C., and Chassignet, E. P.: Lagrangian data in a high-resolution numerical simulation of the North Atlantic, I. Comparison with in situ drifter data, J. Mar. Sys., 29, 157-176, 2001a.

Garraffo, Z. D., Mariano, A. J., Griffa, A., Veneziani, C., and Chassignet, E. P.: Lagrangian data in a high-resolution numerical simulation of the North Atlantic, II. On the pseudo-Eulerian averaging of Lagrangian data, J. Mar. Sys., 29, 177-200, 2001b.

Ivanov, L. M. and Margolina, T. M.: Prediction in geophysical hydrodynamics as the probabilistic problem of the first-passage boundary, In: Proc. Rossby-1000 Symposium, Sweden, 63-69, 1999.

Ivanov, L. M., Kirwan, A. D., Jr., and Melnichenko, O. V.: Prediction of the stochastic behavior of nonlinear systems by determin- 
istic models as a classical time-passage probabilistic problem, Non. Proc. Geophys., 1, 224-233, 1994.

Ivanov, L. M., Margolina, T. M., and Melnichenko, O. V.: Prediction and management of extreme events based on a simple probabilistic model of the first-passage boundary, Phys. Chem. Earth (A), 24, 2, 169-173, 1999.

Ivanov, L. M., Margolina, T. M., McClean, Ju. L., and Poberezhny, Yu. A.: Lagrangian predictability of POP: the special case of North Atlantic, Tellus, (in preparation), 2003.

Jiang, S. and Malanotte-Rizzoli, P.: On the predictability of regional oceanic jet stream: the impact of model errors at the inflow boundary, J. Mar. Res., 57, 641-669, 1999.

Kantha, L. H. and Clayson, C.: Numerical Models of Oceans and Oceanic Processes, Academic Press, 2000.

Kantha, L. H., Choi, J. K., Leben, R., Copper, C., Vogel, M., and Feeney, J.: Hindcasts and real-time nowcast/forecasts of currents in the Gulf of Mexico, Offshore Technology Conference (OTC'99), May 3-6, 1999, Houston, TX, 1999.

Klafer, J., Blumen, A., and Shlesinger, M. F.: Stochastic pathway to anomalous diffusion, Phys. Rev. A, 35, 7, 3081-3085, 1987.

Kravtsov, Yu. A.: Randomness, determinativeness and predictability, Usp. Fiz. Nauk, 158, 93-123, 1989.

Kuznetsov, L., Toner, M., Kirwan, A. D., Jr., Jones, C. K. R. T., Kantha, L. H., and Choi, J.: The Loop Current and adjacent rings delineated by Lagrangian analysis of the near surface flow, J. Mar. Res., 60, 405-429, 2002.

Lacarra, J.-F. and Talagrand, O.: Short range evolution of small perturbations in barotropic model, Tellus, 40A, 81-95, 1988.

Lacorata, G., Aurell, E., and Vulpiani, A.: Drifter dispersion in the Adriatic Sea: Lagrangian data and chaotic model, Ann. Geophys., 19, 121-129, 2001.

Lipphardt, B. L., Jr., Kirwan, A. D., Jr., Grosch, C. E., Lewis, J. K., and Paduan, J. D.: Blending HF radar and model velocities in Monterey Bay through normal mode analysis, J. Geophys. Res., 105, C2, 3425-3450, 2000.

Lorenz, E. N.: A study of the predictability of a 28 -variable atmospheric model, Tellus, 17, 3, 321-333, 1965.

Lorenz, E. H.: Irregularity. A fundamental property of the atmosphere, Tellus, 36, 98-101, 1984.

Mariano, A. J., Griffa, A., Ozgokmen, T. M., and Zambianchi, E.: Lagrangian analysis and predictability of coastal and ocean dynamics 2000, J. Atm. Ocean. Tech., 19, 7, 1114-1126, 2002.

McClean, Ju. L., Poulain, P.-M., and Pelton, J. W.: Eulerian and Lagrangian statistics from surface drifters and a high-resolution simulation in the North Atlantic, J. Phys. Oceanogr., 32, 24722491, 2002

Moffat, H. K.: Magnetic Field Generation in Electrically Conducting Fluids, Cambridge University Press, 1978.

Morse, P. M. and Feshbach, H.: Methods of Theoretical Physics: Part I: chapters 1 to 8, McGraw-Hill Book Co. Inc., 1953.
Nicolis, C.: Probabilistic aspects of error growth in atmospheric dynamics, Q. J. R. Meteorol. Soc., 118, 553-568, 1992.

Palmer, T.: Predicting uncertainty in forecasts of weather and climate, Rep. Prog. Phys., 63, 71-116, 2000.

Penenko, V. V. and Protasov, A. V.: Construction of natural orthogonal bases representing the fields of meteorological elements, Atm. and Ocean Phys., 14, 2, 883-888, 1978.

Pires, C., Vautard, R., and Talagrand, O.: On extending the limits of variational assimilation in nonlinear chaotic systems, Tellus, 48A, 96-121, 1996.

Popov, V. S.: Mean escape time in diffusion, Sov. Phys.-JETP, 31, 4, 750-752, 1970.

Rangarajan, G. and Ding, M.: First passage time distribution for anomalous diffusion, Phys. Lett., A273, 322-330, 2000.

Robinson, A. R., McCarthy, J. J., and Rotschild, B. J.: Interdisciplinary ocean science is evolving and a systems approach is essential, J. Mar. Sys., 22, 231-239, 1999.

Schaudt, K. J., Forristall, G. Z., Kantha, L. H., Lebem, R., Choi, J.K., Black, P., Uhlhom, E., Guinasso, N. L., Jr., Walpert, J. N., Kelly, F. J., DiMarco, S. F., Wang, O., Anderson, S., and Coholon, P.: A look at currents in the Gulf of Mexico in 19992000, Offshore Technology Conference (OTC'01), May 3-6, 2001, Houston, TX, 2001

Schumacher, J. and Eckartdt, B.: Clustering dynamics of lagrangian tracers in free-surface flows, Phys. Rev., E 66, 017303-1017303-14, 2002.

Smith, R. D., Dukowicz, J. K., and Malone, R. C.: Parallel ocean circulation modeling, Physica D, 60, 38-61, 1992.

Stutzer, S. and Krauss, W.: Mean circulation and transports in the South Atlantic Ocean: Combining model and drifter data, J. Geophys. Res., 103 (C13), 30, 985-31,002, 1998.

Syrovich, L.: Chaotic dynamics of coherent structures, Physica D, 37, 126-145, 1989.

Tennekes, H. and Wijgaard, J. C.: The intermittent small-scale structure of turbulence: data processing hazards, J. Fluid Mech., 55, 93-103, 1972.

Toner, M., Kirwan, A. D., Jr., Kantha, L. H., and Choi, J. K.: Can general circulation models be assessed and their output enhanced with drifter data, J. Geophys. Res., 106, C9, 19563-19579, 2001.

Vapnik, V. H.: Reconstruction of Empirical Laws from Observations, Nauka, Moscow (in Russian), 1983

Wiggins, S.: Chaotic Transport in Dynamical Systems, Interdisciplinary Applied Mathematics, Vol. 2., Springer, Berlin, 1992.

Zaslavsky, G. M.: Chaos, fractional kinetics, and anomalous transport, Phys. Rep., 371, 461-580, 2002.

Zeldovich, Ya. B., Ruzmaikin, A.A., and Sokolov, V. V.: Representation of a three dimensional vector field by scalar potentials, Sov. Phys. Doklady, 30, 756-758. 1985. 